\title{
AN INTRODUCTION TO PRESSURE METRICS FOR HIGHER TEICHMÜLLER SPACES
}

\author{
MARTIN BRIDGEMAN, RICHARD CANARY, AND ANDRÉS SAMBARINO
}

\section{CONTENTS}

1. Introduction

2. The Thermodynamic Formalism 2

3. Basic strategy 6

4. The pressure metric for Teichmüller space 8

5. The pressure metric on the Hitchin component 12

6. Generalizations and consequences 26

7. Open problems 32

References $\quad 35$

\section{INTRODUCTION}

We discuss how one uses the Thermodynamic Formalism to produce metrics on higher Teichmüller spaces. Our higher Teichmüller spaces will be spaces of Anosov representations of a word hyperbolic group into a semisimple Lie group. To each such representation we associate an Anosov flow encoding eigenvalue information, and the Thermodynamic Formalism gives us a way to measure the difference between two such flows. This difference gives rise to an analytic semi-norm, which in many cases turns out to be a Riemannian metric, called the pressure metric. This paper surveys results of Bridgeman-Canary-Labourie-Sambarino [17] and discusses questions and open problems which arise.

We begin by discussing our construction in the classical setting of the Teichmüller space of a closed orientable surface of genus at least 2. In this setting, our construction agrees with Thurston's Riemannian metric, as reinterpreted by Bonahon [8] using geodesic currents and McMullen [62] using the Thermodynamic Formalism. Wolpert [90] showed that Thurston's metric

Bridgeman was partially supported by NSF grant DMS - 1500545, Canary was partially supported by NSF grant DMS - 1306992 and Sambarino was partially supported by the European Research Council under the European Community's seventh Framework Programme (FP7/2007-2013)/ERC grant agreement $\mathrm{n}^{\circ}$ FP7-246918. The authors also acknowledge support from U.S. National Science Foundation grants DMS 1107452, 1107263, 1107367 "RNMS: GEometric structures And Representation varieties" (the GEAR Network). 
is a multiple of the Weil-Petersson metric. The key difference between our approach and McMullen's is that we work directly with the geodesic flow of the surface, rather than with Bowen-Series coding of the action of the group on the limit set. Since such a coding is not known to exist for every hyperbolic group, this approach will be crucial to generalizing our results to the setting of all hyperbolic groups.

We next discuss the construction of the pressure metric in the simplest new situation: the Hitchin component of representations of a surface group into $P S L_{d}(\mathbb{R})$. This setting offers the cleanest results and also several simplifications of the general proof. Given a Hitchin representation, inspired by earlier work of Sambarino [74], we construct a metric Anosov flow, which we call the geodesic flow of the representation, whose periods record the spectral radii of the elements in the image. We obtain a mapping class group invariant Riemannian metric on a Hitchin component whose restriction to the Fuchsian locus is a multiple of the Weil-Petersson metric.

We hope that the discussion of the pressure metric in these two simpler settings will provide motivation and intuition for the general construction. In section 6 we discuss the more general settings studied in [17] with some comments on the additional difficulties which must be overcome. We finish with a discussion of open problems.

Acknowledgements: The paper is based on a Master Class given by the authors at the Centre for Quantum Geometry of Moduli Spaces in Aarhus. We thank Jorgen Anderson for the invitation to give this Master Class and the editors for the invitation to write this article. We thank Marc Burger, François Labourie and Adam Sikora for helpful conversations. Substantial portions of this paper were written while the authors were in residence at the Mathematical Sciences Research Institute in Berkeley, CA, during the Spring 2015 semester and were partially supported by NSF grant No. 0932078000.

\section{The Thermodynamic Formalism}

The Thermodynamic Formalism was introduced by Bowen and Ruelle $([11,12,73])$ as a tool to study the ergodic theory of Anosov flows and diffeomorphisms. It was further developed by Parry and Pollicott, their monograph [66] is a standard reference for the material covered here. McMullen [62] introduced the pressure form as a tool for constructing metrics on spaces which may be mapped into Hölder potentials over a shift-space. We will give a quick summary of the basic facts we will need, but we en-

courage the reader to consult the original references and the more complete discussion and references in [17].

We recall that a smooth flow $\phi=\left(\phi_{t}: X \rightarrow X\right)_{t \in \mathbb{R}}$ on a compact Riemannian manifold is said to be Anosov if there is a flow-invariant splitting $T X=E^{s} \oplus E_{0} \oplus E^{u}$ where $E_{0}$ is a line bundle parallel to the flow and if $t>0$, then $d \phi_{t}$ is exponentially contracting on $E_{+}$and $d \phi_{-t}$ is exponentially contracting on $E_{-}$. We will always assume that our Anosov flows are 
topologically transitive (i.e. have a dense orbit). It is a celebrated theorem of Anosov (see [48, Thm. 17.5.1]) that the geodesic flow of a closed hyperbolic surface, and more generally of a closed negatively curved manifold, is a topologically transitive Anosov flow.

2.1. Entropy, pressure and orbit-equivalence. Let $\phi$ be a topologically transitive Anosov flow on a compact Riemannian manifold $X$. If $a$ is a $\phi$ periodic orbit, denote by $\ell(a)$ its period and let

$$
R_{T}=\{a \text { closed orbit } \mid \ell(a) \leqslant T\} .
$$

Then, following Bowen [9], we may define the topological entropy of $\phi$ to be the exponential growth rate of the number of periodic orbits whose periods are at most $T$, i.e.

$$
h(\phi)=\limsup _{T \rightarrow \infty} \frac{\log \# R_{T}}{T} .
$$

Moreover, if $g: X \rightarrow \mathbb{R}$ is Hölder and $a$ is a closed orbit, denote by

$$
\ell_{g}(a)=\int_{0}^{\ell(a)} g\left(\phi_{s}(x)\right) d s
$$

where $x$ is any point on $a$. Then, following Bowen-Ruelle [12], we may define the topological pressure of $g$ (or simply pressure) by

$$
\mathbf{P}(g)=\mathbf{P}(\phi, g)=\limsup _{T \rightarrow \infty} \frac{1}{T} \log \left(\sum_{a \in R_{T}} e^{\ell_{g}(a)}\right) .
$$

Note that $\mathbf{P}(g)$ only depends on the periods of $g$, i.e. the collection of numbers $\left\{\ell_{g}(a)\right\}$.

Livšic provides a pointwise relation for two functions having the same periods: two Hölder functions $f, g: X \rightarrow \mathbb{R}$ are Livšic cohomologous if there exists a Hölder function $V: X \rightarrow \mathbb{R}$, which is $C^{1}$ in the direction of the flow $\phi$, such that

$$
f(x)-g(x)=\left.\frac{\partial}{\partial t}\right|_{t=0} V\left(\phi_{t}(x)\right) .
$$

Livšic [55] proved the following fundamental result:

Theorem 2.1 (Livšic [55]). If $\phi$ is a topologically transitive Anosov flow and $g: X \rightarrow \mathbb{R}$ is a Hölder function such that $\ell_{g}(a)=0$ for every closed orbit a, then $g$ is Livšic cohomologous to 0 .

Given a positive Hölder function $f: X \rightarrow(0, \infty)$ one may define a reparametrization of the flow so that its "speed" at a point $x$ is multiplied by $f(x)$. More formally, let

$$
\kappa_{f}(x, t)=\int_{0}^{t} f\left(\phi_{s}(x)\right) d s,
$$


and define $\phi^{f}=\left(\phi_{t}^{f}: X \rightarrow X\right)_{t \in \mathbb{R}}$ so that $\phi_{\kappa_{f}(x, t)}^{f}(x)=\phi_{t}(x)$. In particular, if $a$ is a $\phi$-closed orbit then $a$ is also a closed orbit of the flow $\phi^{f}$ with period $\ell_{f}(a)$.

A Hölder orbit equivalence between two flows is a Hölder homeomorphism that sends orbits to orbits. Moreover, if it preserves time, it is called a Hölder conjugacy. In particular, the identity map is a Hölder orbit equivalence from $\phi$ to $\phi^{f}$ when $f$ is a positive Hölder function. (In our setting, the inverse of any Hölder orbit equivalence is also a Hölder orbit equivalence, but we will not need this fact.)

Livšic's theorem implies that two positive Hölder functions are Livšic cohomologous if and only if the periods of $\phi^{f}$ and $\phi^{g}$ agree. If this is the case, the function $V$ in Equation (1) provides a Hölder conjugacy between $\phi^{f}$ and $\phi^{g}$. Moreover, one has the following standard consequence of Livšic's Theorem (see Sambarino [75, Lemma 2.11]).

Lemma 2.2. If $\phi$ is a topologically transitive, Anosov flow and there exists Hölder orbit equivalence to a Hölder-continuous flow $\psi$, then there exists a Hölder function $f: X \rightarrow(0, \infty)$ such that $\psi$ is Hölder conjugate to $\phi^{f}$.

The flow $\phi^{f}$ remains topologically transitive and is again Anosov but in a metric sense. More specifically, $\phi^{f}$ is a Smale flow in the sense of Pollicott [68]. Pollicott shows that all the results we rely on in the ensuing discussion generalize to the setting of Smale flows, which we will refer to as metric Anosov flows. Hence, we may define the topological entropy of $\phi^{f}$ as

$$
h(f)=\limsup _{T \rightarrow \infty} \frac{\log \# R_{T}(f)}{T},
$$

where $R_{T}(f)=\left\{a\right.$ closed orbit $\left.\mid \ell_{f}(a) \leqslant T\right\}$. We recall the following standard lemma which relates pressure and entropy.

Lemma 2.3. (see Sambarino [74, Lemma 2.4]) If $\phi$ is a topologically transitive Anosov flow and $f: X \rightarrow(0, \infty)$ is Hölder, then $\mathbf{P}(-h f)=0$ if and only if $h=h(f)$.

Ruelle [73, Cor. 7.10] (see also Parry-Pollicott [66, Prop. 4.7]) proved that the pressure function $\mathbf{P}(g)$ is a real analytic function of the Hölder function $\mathrm{g}$. It follows from Lemma 2.3 and the Implicit Function Theorem that entropy varies analytically in $f$. Ruelle [72] used a similar observation to show that the Hausdorff dimension of a quasifuchsian Kleinian group varies analytically on quasifuchsian space.

Ruelle [73] also showed that $\mathbf{P}$ is a convex function and thus if $f: X \rightarrow \mathbb{R}$ and $g: X \rightarrow \mathbb{R}$ are Hölder functions,

Consider the space

$$
\left.\frac{\partial^{2}}{\partial t^{2}}\right|_{t=0} \mathbf{P}(f+t g) \geqslant 0
$$

$$
\mathcal{P}(X)=\{\Phi: X \rightarrow \mathbb{R} \text { Hölder } \mid \mathbf{P}(\Phi)=0\}
$$


of pressure zero Hölder functions on $X$. It follows immediately from the definition above that the pressure function $\mathbf{P}$ is constant on Livšic cohomology classes, so it is natural to consider the space

$$
\mathcal{H}(X)=\mathcal{P}(X) / \sim
$$

of Livšic cohomology classes of pressure zero functions.

McMullen [62] defined a pressure semi-norm on the tangent space of the space of pressure zero Hölder functions on a shift space. Similarly, we define a pressure semi-norm on $\mathrm{T}_{f} \mathcal{P}(X)$, by letting

$$
\|g\|_{\mathbf{P}}^{2}=\left(\left.\frac{\partial^{2}}{\partial t^{2}}\right|_{t=0} \mathbf{P}(f+t g)\right)\left(\frac{-1}{\left.\frac{\partial}{\partial t}\right|_{t=0} \mathbf{P}(f+t f)}\right)
$$

for all $g \in \mathrm{T}_{f} \mathcal{P}(X)=\operatorname{ker} D_{f} \mathbf{P}$. (Formally, one should consider the space $\mathcal{P}^{\alpha}(X)$ of $\alpha$-Hölder pressure zero functions for some $\alpha>0$. In all our applications, we will consider embeddings of analytic manifolds into $\mathcal{P}(X)$ such that every point has a neighborhood which maps into $\mathcal{P}^{\alpha}(X)$ for some $\alpha>0$. We will consistently suppress this technical detail.)

One obtains the following characterization of degenerate vectors, due to Ruelle and Parry-Pollicott.

Theorem 2.4. (Ruelle [73], see also Parry-Pollicott [66, Prop. 4.12]) Let $\phi$ be a topologically transitive Anosov flow and consider $g \in \mathrm{T} \mathcal{P}(X)$. Then, $\|g\|_{\mathbf{P}}=0$ if and only if $g$ is Livšic cohomologous to zero, i.e. $\ell_{g}(a)=0$ for every closed orbit a.

We make use of the following nearly immediate corollary of this characterization (see the proof of [17, Lemma 9.3]).

Corollary 2.5. Let $\phi$ be a topologically transitive Anosov flow. Suppose that $\left\{f_{t}\right\}_{t \in(-1,1)}: X \rightarrow(0, \infty)$ is a smooth one parameter family of Hölder functions. Consider $\Phi:(-1,1) \rightarrow \mathcal{P}(X)$ defined by $\Phi(t)=-h\left(f_{t}\right) f_{t}$. Then $\left\|\dot{\Phi}_{0}\right\|_{\mathbf{P}}=0$ if and only if

$$
\left.\frac{\partial}{\partial t}\right|_{t=0} h\left(f_{t}\right) \ell_{f_{t}}(a)=0
$$

for every closed orbit a of $\phi$.

2.2. Intersection and Pressure form. Inspired by Bonahon's [8] intersection number, we define the intersection number $\mathbf{I}$ of two positive Hölder functions $f_{1}, f_{2}: X \rightarrow(0, \infty)$ by

$$
\mathbf{I}\left(f_{1}, f_{2}\right)=\lim _{T \rightarrow \infty} \frac{1}{\# R_{T}\left(f_{1}\right)} \sum_{a \in R_{T}\left(f_{1}\right)} \frac{\ell_{f_{2}}(a)}{\ell_{f_{1}}(a)}
$$

and their renormalized intersection number by

$$
\mathbf{J}\left(f_{1}, f_{2}\right)=\frac{h\left(f_{2}\right)}{h\left(f_{1}\right)} \mathbf{I}\left(f_{1}, f_{2}\right) .
$$


Bowen's equidistribution theorem on periodic orbits [9] implies that I, and hence $\mathbf{J}$, are well-defined (see [17, Section 3.4]). One may use the analyticity of the pressure function and results of Parry-Pollicott [66] and Ruelle [73] to check that they are analytic functions.

Proposition 2.6. ([17, Prop. 3.12]) Let $\phi$ be a topologically transitive Anosov flow and let $\left\{f_{u}\right\}_{u \in M}$ and $\left\{g_{u}\right\}_{u \in M}$ be two analytic familes of positive Hölder functions on $X$. Then $h\left(f_{u}\right)$ varies analytically over $M$ and $\mathbf{I}\left(f_{u}, g_{u}\right)$ and $\mathbf{J}\left(f_{u}, g_{u}\right)$ vary analytically over $M \times M$.

The seminal work of Bowen and Ruelle [12] may be used to derive the following crucial rigidity property for the renormalized intersection number.

Proposition 2.7. ([17, Prop. 3.8]) If $\phi$ is a topologically transitive Anosov flow on $X$ and $f$ and $g$ are positive Hölder functions on $X$, then

$$
\mathbf{J}(f, g) \geq 1 .
$$

Moreover, $\mathbf{J}(f, g)=1$ if and only if $h(f) f$ and $h(g) g$ are Livšic cohomologous.

If $\left\{f_{u}\right\}_{u \in M}$ is an analytic family of positive Hölder functions on $X$, then, for all $u \in M$, we consider the function

$$
\mathbf{J}_{u}: M \rightarrow \mathbb{R}
$$

given by $\mathbf{J}_{u}(v)=\mathbf{J}(u, v)$ for all $v \in M$. Proposition 2.7 implies that the Hessian of $J_{u}$ gives a non-negative bilinear form on $T_{u} M$. Lemma 2.3 allows us to define a thermodynamic mapping

$$
\Phi: M \rightarrow \mathcal{P}(X)
$$

by letting $\Phi(u)=-h\left(f_{u}\right) f_{u}$ The following important, but fairly simple, result shows that this bilinear form is the pull-back of the pressure form.

Proposition 2.8. ([17, Prop. 3.11]) Let $\phi$ be a topologically transitive Anosov flow. If $\left\{f_{t}\right\}_{t \in(-1,1)}$ is a smooth one parameter family of positive Hölder functions on $X$ and $\Phi:(-1,1) \rightarrow \mathcal{P}(X)$ is given by $\Phi(t)=-h\left(f_{t}\right) f_{t}$, then

$$
\left\|\dot{\Phi}_{0}\right\|_{\mathbf{P}}^{2}=\left.\frac{\partial^{2}}{\partial t^{2}}\right|_{t=0} \mathbf{J}\left(f_{0}, f_{t}\right)
$$

\section{BASIC STRATEGY}

Our basic strategy is inspired by McMullen's [62] re-interpretation of Thurston's Riemannian metric on Teichmüller space and its generalization to quasifuchsian space by Bridgeman [14].

We consider a family $\left\{\rho_{u}: \Gamma \rightarrow \mathrm{G}\right\}_{u \in M}$ of (conjugacy classes of ) representations of a word hyperbolic group $\Gamma$ into a semi-simple Lie group $G$ parametrized by an analytic manifold $M$. We recall that Gromov [36] associated a geodesic flow $\phi=\left\{\phi_{t}: U_{\Gamma} \rightarrow U_{\Gamma}\right\}_{t \in \mathbb{R}}$ to a hyperbolic group $\Gamma$ which 
agrees with the geodesic flow on $T^{1} X$ in the case when $\Gamma$ is the fundamental group of a negatively curved manifold $X$ (see Section 6 for details).

In our two basic examples $\Gamma=\pi_{1}(S)$ where $S$ is a closed, oriented surface of genus at least 2 , and the flow $\phi$ is the geodesic flow on a hyperbolic surface homeomorphic to $S$. The first example will be the classical Teichmüller space $\mathcal{T}(S)$ of hyperbolic structures on $S$, where $\mathrm{G}=\mathrm{PSL}_{2}(\mathbb{R})$ and $M=\mathcal{T}(S)$. The second is the Hitchin component $\mathcal{H}_{d}(S)$, where $\mathrm{G}=\mathrm{PSL}_{\mathrm{d}}(\mathbb{R})$ and $M=\mathcal{H}_{d}(S)$.

Step 1: Associate to each representation $\rho_{u}$ a topologically transitive metric Anosov flow $\phi^{\rho_{u}}$ which is Hölder orbit equivalent to the geodesic flow $\phi$ of $\Gamma$ so that the period of the orbit associated to $\gamma \in \Gamma$ is the "length" of $\rho(\gamma)$.

In the case of $\mathcal{T}(S), \phi^{\rho}$ will be the geodesic flow of the surface $X_{\rho}=$ $\mathbb{H}^{2} / \rho(S)$. In the case of a Hitchin component, we will construct a geodesic flow and our notion of length will be the logarithm of the spectral radius.

If $u \in M$, Lemma 2.2 provides a positive Hölder function $f_{u}: U_{\Gamma} \rightarrow \mathbb{R}$, well-defined up to Livšic cohomology, such that $\phi^{\rho_{u}}$ is Hölder conjugate to $\phi^{f_{u}}$.

Step 2: Define a thermodynamic mapping $\Phi: M \rightarrow \mathcal{H}\left(U_{\Gamma}\right)$ by letting $\Phi(u)=\left[-h\left(f_{u}\right) f_{u}\right]$ and prove that it has locally analytic lifts, i.e. if $u \in M$, then there exists a neighborhood $U$ of $u$ in $M$ and an analytic map $\tilde{\Phi}: U \rightarrow \mathcal{P}\left(U_{\Gamma}\right)$ which is a lift of $\left.\Phi\right|_{U}$.

We may also define a renormalized intersection number on $M \times M$, by letting $\mathbf{J}(u, v)=\mathbf{J}\left(f_{u}, f_{v}\right)$.

Step 3: Define a pressure form on $M$ by pulling back the pressure from on $\mathcal{P}\left(U_{\Gamma}\right)$ by (the lifts of) $\Phi$.

Lemma 2.8 allows us to reinterpret the pull-back of the pressure form as the Hessian of the renormalized intersection number function.

Step 4: Prove, using Corollary 2.5, that the resulting pressure form is nondegenerate so gives rise to an analytic Riemannian metric on $M$.

Step 4 can fail in certain situations. For example, Bridgeman's pressure metric on quasifuchsian space [14] is degenerate exactly on the set of pure bending vectors on the Fuchsian locus. However, Bridgeman's pressure metric still gives rise to a path metric.

Historical remarks: Thurston's constructed a Riemannian metric which he describes as the "Hessian of the length of a random geodesic." Wolpert's formulation [90] of this construction agrees with the Hessian of the intersection number of the geodesic flows. From this viewpoint, one regards $\mathbf{I}(\rho, \eta)$, as the length in $X_{\eta}$ of a random unit length geodesic on $X_{\rho}$. If one considers a sequence $\left\{\gamma_{n}\right\}$ of closed geodesics on $X_{\rho}$ which are becoming equidistributed (in the sense that $\left\{\frac{\gamma_{n}}{\ell_{\rho}\left(\gamma_{n}\right)}\right\}$ converges, in the space of geodesic currents on $S$, 
to the Liouville current $\nu_{\rho}$ of $X_{\rho}$ ), then

$$
\mathbf{I}\left(f_{\rho}, f_{\eta}\right)=\lim \frac{\ell_{\eta}\left(\gamma_{n}\right)}{\ell_{\rho}\left(\gamma_{n}\right)} .
$$

Bonahon [8] reinterprets this to say that

$$
\mathbf{I}\left(f_{\rho}, f_{\eta}\right)=i\left(\nu_{\rho}, \nu_{\eta}\right)
$$

where $i$ is the geometric intersection pairing on the space of geodesic currents.

Bridgeman and Taylor [18] used Patterson-Sullivan theory to show that the Hessian of the renormalized intersection number is a non-negative form on quasifuchsian case. McMullen [62] then introduced the use of the techniques of Thermodynamic Formalism to interpret both of these metrics as pullbacks of the pressure metric on the space of suspension flows on the shift space associated to the Bowen-Series coding. Bridgeman [14] then showed that the resulting pressure form on quasifuchsian space is degenerate exactly on the set of pure bending vectors on the Fuchsian locus.

\section{The PRessure Metric FOR TeIChMÜller SPACE}

In this section, we survey the construction of the pressure metric for the Teichmüller space $\mathcal{T}(S)$ of a closed oriented surface $S$ of genus $g \geq 2$.

We recall that $\mathcal{T}(S)$ may be defined as the unique connected component of

$$
\operatorname{Hom}\left(\pi_{1}(S), \mathrm{PSL}_{2}(\mathbb{R})\right) / \mathrm{PGL}_{2}(\mathbb{R})
$$

which consists of discrete and faithful representations. If $\rho \in \mathcal{T}(S)$, then one obtains a hyperbolic surface $X_{\rho}=\mathbb{H}^{2} / \rho\left(\pi_{1}(S)\right)$ by regarding $\mathrm{PSL}_{2}(\mathbb{R})$ as the space of orientation-preserving isometries of the hyperbolic plane $\mathbb{H}^{2}$.

4.1. Basic facts. It is useful to isolate the facts that will make the construction much simpler in this case. All of these facts will fail even in the setting of the Hitchin component.

(1) The space $T^{1} \mathbb{H}^{2}$ is canonically identified with the space of ordered triplets on the visual boundary $\partial_{\infty} \mathbb{H}^{2}$,

$$
\left(\partial_{\infty} \mathbb{H}^{2}\right)^{(3)}=\left\{(x, y, z) \in\left(\partial_{\infty} \mathbb{H}^{2}\right)^{3}: x<y<z\right\},
$$

where $<$ is defined by a given orientation on the topological circle $\partial_{\infty} \mathbb{H}^{2}$, and $(x, y, z)$ is identified with the unit tangent vector to the geodesic $L$ joining $x$ to $z$ at the point which is the orthogonal projection of $y$ to $L$.

(2) The surface $X_{\rho}$ is closed (since it is a surface homotopy equivalent to a closed surface). In fact, by Baer's Theorem, it is diffeomorphic to $S$. The geodesic flow $\phi^{\rho}$ on $\mathrm{T}^{1} X_{\rho}$ is thus a topologically transitive Anosov flow on a closed manifold.

(3) The topological entropy $h(\rho)$ of $\phi^{\rho}$ is equal to 1 (in particular, constant). 
Fact (1) is quite straight-forward: if $(p, v) \in \mathrm{T}^{1} \mathbb{H}^{2}$, denote by $v_{\infty} \in \partial_{\infty} \mathbb{H}^{2}$ the limit at $+\infty$ of the geodesic ray starting at $(p, v)$, then the identification is

$$
(p, v) \mapsto\left((-v)_{\infty},(i v)_{\infty}, v_{\infty}\right)
$$

where $i v \in \mathrm{T}^{1} \mathbb{H}^{2}$ is such that the base $\{v, i v\}$ is orthogonal and oriented.

Fact (3) is a standard consequence of the fact, due to Manning [58], that the entropy of the geodesic flow of a negatively curved manifold agrees with the exponential rate of volume growth of a ball of radius $T$ in its universal cover. In this setting, the universal cover is always $\mathbb{H}^{2}$ so the entropy is always 1 .

Conventions: For the remainder of the section we fix $\rho_{0} \in \mathcal{T}(S)$ and identify $S$ with $X_{\rho_{0}}$. We then obtain an identification of $\partial_{\infty} \pi_{1}(S)$ with $\partial_{\infty} \mathbb{H}^{2}$ and of $\mathrm{T}^{1} S$ with $\mathrm{T}^{1} X_{\rho_{0}}$. Let $\phi=\phi^{\rho_{0}}$ be the geodesic flow on $S$.

It will be useful to choose an analytic lift $s: \mathcal{T}(S) \rightarrow \operatorname{Hom}\left(\pi_{1}(\mathrm{~S}), \mathrm{PSL}_{2}(\mathbb{R})\right)$. In order to do so, we pick non-commuting elements $\alpha$ and $\beta$ in $\pi_{1}(S)$ and choose a representative $\rho=s([\rho])$ of $[\rho]$ such that $\rho(\alpha)$ has attracting fixed point $+\infty \in \partial_{\infty} \mathbb{H}^{2}$ and repelling fixed point 0 , while $\rho(\beta)$ has attracting fixed point 1. From now on, we will implicitly identify $\mathcal{T}(S)$ with $s(\mathcal{T}(S)$ ). This choice will allow us to define our thermodynamic mapping into the space $\mathcal{P}\left(\mathrm{T}^{1} S\right)$ of pressure zero Hölder functions on $\mathrm{T}^{1} S$, rather than just into the space $\mathcal{H}\left(\mathrm{T}^{1} S\right)$ of Livšic cohomology classes of pressure zero Hölder functions on $X$.

4.2. Analytic variation of limit maps. It is well known that any two Fuchsian representations are conjugate by a unique Hölder map.

Proposition 4.1. If $\rho, \eta \in \mathcal{T}(S)$, then there is a unique $(\rho, \eta)$-equivariant Hölder homeomorphism $\xi_{\rho, \eta}: \partial_{\infty} \mathbb{H}^{2} \rightarrow \partial_{\infty} \mathbb{H}^{2}$. Moreover, $\xi_{\rho, \eta}$ varies analytically in $\eta$.

Proof. By fact (2), there exists a diffeomorphism $h: X_{\rho} \rightarrow X_{\eta}$ in the homotopy class determined by $\eta \circ \rho^{-1}$. Choose a $(\rho, \eta)$-equivariant lift $\tilde{h}: \mathbb{H}^{2} \rightarrow \mathbb{H}^{2}$ of $h$. Since $\tilde{h}$ is quasiconformal, classical results in complex analysis (see Ahlfors-Beurling [1]), imply that $\tilde{h}$ extends to a quasisymmetric map $\xi_{\rho, \eta}: \partial_{\infty} \mathbb{H}^{2} \rightarrow \partial_{\infty} \mathbb{H}^{2}$. In particular, $\xi_{\rho, \eta}$ is a Hölder homeomorphism. Since $\tilde{h}$ is $(\rho, \eta)$-equivariant, so is $\xi$. The resulting map is unique, since, by equivariance, if $\gamma \in \pi_{1}(S)$, then $\xi_{\rho, \eta}$ must take the attracting fixed point of $\rho(\gamma)$ to the attracting fixed point of $\eta(\gamma)$.

A more modern approach to the existence of $\xi_{\rho, \eta}$ uses the fact that $\mathbb{H}^{2}$ is a proper hyperbolic metric space with boundary $\partial_{\infty} \mathbb{H}^{2}$ and that quasiisometries of proper hyperbolic metric spaces extend to Hölder homeomorphisms of their boundary. Since $h$ is a bilipschitz homeomorphism, it lifts to a bilipschitz homeomorphism of $\mathbb{H}^{2}$. In particular, $\tilde{h}$ is a quasi-isometry of $\mathbb{H}^{2}$. 
It is a classical result in Teichmüller theory that $\xi_{\rho, \eta}$ varies analytically in $\eta$. A more modern, but still complex analytic, approach uses holomomorphic motions and is sketched by McMullen [62, Section 2]. One allows $\eta$ to vary over the space $Q F(S)$ of (conjugacy classes of) convex cocompact (i.e. quasifuchsian) representations of $\pi_{1}(S)$ into $\mathrm{PSL}_{2}(\mathbb{C})$. (Recall that $Q F(S)$ is an open neighborhood of $\mathcal{T}(S)$ in the $\mathrm{PSL}_{2}(\mathbb{C})$-character variety of $\pi_{1}(S)$.) If $\eta \in Q F(S)$, there is a $(\rho, \eta)$-equivariant embedding $\xi_{\rho, \eta}: \partial_{\infty} \mathbb{H}^{2} \rightarrow \widehat{\mathbb{C}}$ whose image is the limit set of $\eta\left(\pi_{1}(S)\right)$. If $z \in \partial_{\infty} \mathbb{H}^{2}$ is a fixed point of a nontrivial element $\rho(\gamma)$, then $\xi_{\rho, \eta}(z)$ varies holomorphically in $\eta$. Slodkowski's generalized Lambda Lemma [79] then implies that $\xi_{\rho, \eta}$ varies complex analytically as $\eta$ varies over $Q F(S)$, and hence varies real analytically as $\eta$ varies over $\mathcal{T}(S)$.

One may also prove analyticity by using techniques of Hirsch-Pugh-Shub [41] as discussed in the next section.

4.3. The thermodynamic mapping. The next proposition allows us to construct the thermodynamic mapping we use to define the pressure metric.

Proposition 4.2. For every $\eta \in \mathcal{T}(S)$, there exists a positive Hölder function $f_{\eta}: \mathrm{T}^{1} S \rightarrow(0, \infty)$ such that

$$
\int_{[\gamma]} f_{\eta}=\ell_{\eta}(\gamma)
$$

for all $\gamma \in \pi_{1}(S)$. Moreover, $f_{\eta}$ varies analytically in $\eta$.

Proof. Let $\xi_{\rho_{0}, \eta}$ be the $\left(\rho_{0}, \eta\right)$-equivariant map provided by Proposition 4.1. The identification of $\mathrm{T}^{1} \mathbb{H}^{2}$ with $\partial_{\infty} \pi_{1}(S)^{(3)}$ gives a $\left(\rho_{0}, \eta\right)$-equivariant Hölder homeomorphism $\tilde{\sigma}: \mathrm{T}^{1} \mathbb{H}^{2} \rightarrow \mathrm{T}^{1} \mathbb{H}^{2}$ defined by

$$
\tilde{\sigma}(x, y, z)=\left(\xi_{\rho_{0}, \eta}(x), \xi_{\rho_{0}, \eta}(y), \xi_{\rho_{0}, \eta}(z)\right) .
$$

Since $\tilde{\sigma}$ is a $\left(\rho_{0}, \eta\right)$-equivariant map sending geodesics to geodesics, the quotient $\sigma: \mathrm{T}^{1} S \rightarrow \mathrm{T}^{1} X_{\eta}$ is a Hölder orbit equivalence between the geodesic flows $\phi=\phi^{\rho_{0}}$ and $\phi^{\eta}$. Lemma 2.2 gives the existence of a function $f_{\eta}$, but in order to establish the analytic variation we give an explicit construction.

If $a, b, c, d \in \partial_{\infty} \mathbb{H}^{2}$, then the signed-distance between the orthogonal projections of $b$ and $c$ onto the geodesic with endpoints $a$ and $b$ is $\log |B(a, b, c, d)|$ where

$$
B(a, b, c, d)=\frac{(a-c)(a-d)}{(b-d)(b-c)}
$$

is the cross-ratio. Let

$$
\kappa_{\rho, \eta}((x, y, z), t)=\log \left(B \left(\xi_{\rho_{0}, \eta}(x), \xi_{\rho_{0}, \eta}(z), \xi_{\rho_{0}, \eta}(y), \xi_{\rho_{0}, \eta}\left(u_{t}(x, y, z)\right)\right.\right.
$$

where $u_{t}$ is determined by $\phi_{t}^{\rho_{0}}(x, y, z)=\left(x, u_{t}(x, y, z), z\right)$. We average $\kappa_{\rho, \eta}$ over intervals of length one in the flow to obtain

$$
\kappa_{\rho_{0}, \eta}^{1}((x, y, z), t)=\int_{0}^{1} \kappa_{\rho_{0}, \eta}((x, y, z), t+s) d s
$$


Then

$$
f_{\eta}(x, y, z)=\left.\frac{\partial}{\partial t}\right|_{t=0} \kappa_{\rho_{0}, \eta}^{1}((x, y, z), t)
$$

is Hölder and varies analytically in $\eta$.

One may also prove the analyticity of the reparametrizations in this setting using the techniques of Katok-Knieper-Pollicott-Weiss [49].

Since $h\left(\phi^{\eta}\right)=h\left(f_{\eta}\right)=1$, by Fact (3) in Section 4.1, Lemma 2.3 implies that $\mathbf{P}\left(-f_{\eta}\right)=0$, where $\mathbf{P}$ is the pressure function associated to the geodesic flow $\phi^{\rho_{0}}$ on our base surface $S=X_{\rho_{0}}$. Hence, Proposition 4.2 provides an analytic map $\Phi: \mathcal{T}(S) \rightarrow \mathcal{P}\left(\mathrm{T}^{1} S\right)$ from the Teichmüller space $\mathcal{T}(S)$ to the space $\mathcal{P}\left(\mathrm{T}^{1} S\right)$ of pressure zero functions on the unit tangent bundle $\mathrm{T}^{1} S$, given by

$$
\Phi(\eta)=-f_{\eta}
$$

We call $\Phi$ the thermodynamic mapping. We note that $\Phi$ depends on our choice of $\rho_{0} \in \mathcal{T}(S)$ and on the lift $s: \mathcal{T}(S) \rightarrow \operatorname{Hom}\left(\pi_{1}(S), \mathrm{PSL}_{2}(\mathbb{R})\right)$.

4.4. The pressure metric. We may then define a pressure form on $\mathcal{T}(S)$ by pulling back the pressure form on $\mathcal{P}\left(\mathrm{T}^{1} S\right)$. Explicitly, if $\left\{\eta_{t}\right\}_{t \in(-1,1)}$ is an analytic path in $\mathcal{T}(S)$, then we define

$$
\left\|\dot{\eta}_{0}\right\|_{\mathbf{P}}^{2}=\left\|d \Phi\left(\dot{\eta}_{0}\right)\right\|_{\mathbf{P}}^{2}
$$

Proposition 2.8 will allow us to identify the pressure form with the Hessian of the intersection number $\mathbf{I}$.

Theorem 4.3. (Thurston, Wolpert [90], McMullen [62]) The pressure form is an analytic Riemannian metric on $\mathcal{T}(S)$ which is invariant under the mapping class group and independent of the reference metric $\rho_{0}$. Moreover, the resulting pressure metric is a constant multiple of the Weil-Petersson metric on $\mathcal{T}(S)$.

Proof. We first show that the pressure form is non-degenerate, so gives rise to a Riemannian metric. Consider an analytic path $\left\{\eta_{t}\right\}_{(-1,1)} \subset \mathcal{T}(S)$. If $\left\|d \Phi\left(\dot{\eta}_{0}\right)\right\|_{\mathbf{P}}=0$, then Lemma 2.5 implies that if $\gamma \in \pi_{1}(S)$, then

$$
\left.\frac{\partial}{\partial t}\right|_{t=0} \ell_{\eta t}(\gamma)=0
$$

However, there exist $6 g-5$ elements $\left\{\gamma_{1}, \ldots, \gamma_{6 g-5}\right\}$ of $\pi_{1}(S)$, so that the mapping from $\mathcal{T}(S)$ into $\mathbb{R}^{6 g-5}$ given by taking $\rho$ to $\left(\ell_{\rho}\left(\gamma_{i}\right)\right)_{i=1}^{6 g-5}$ is a real analytic embedding (see Schmutz [77]). Therefore, since $\left.\frac{\partial}{\partial t}\right|_{t=0} \ell_{\eta_{t}}\left(\gamma_{i}\right)=0$ for all $i$, we conclude that $\dot{\eta}_{0}=0$. Therefore, the pressure form is nondegenerate.

If $\rho, \eta \in \mathcal{T}(S)$, the intersection number

$$
\mathbf{I}(\rho, \eta)=\mathbf{I}\left(f_{\rho}, f_{\eta}\right)=\lim _{T \rightarrow \infty} \frac{1}{\# R_{T}(\rho)} \sum_{[\gamma] \in R_{T}(\rho)} \frac{\ell_{\sigma}(\gamma)}{\ell_{\rho}(\gamma)}
$$


where $\ell_{\rho}(\gamma)$ is the translation length of $\rho(\gamma)$ and $R_{T}(\rho)$ is the collection of conjugacy classes of elements of $\pi_{1}(S)$ whose images have translation length at most $T$. So, $\mathbf{I}$ is independent of the reference metric $\rho_{0}$ and invariant by the action of the mapping class group of $S$. Proposition 2.8 states that

$$
\left\|\dot{\eta}_{0}\right\|_{\mathbf{P}}=\left.\frac{\partial^{2}}{\partial t^{2}}\right|_{t=0} \mathbf{J}\left(f_{\eta_{0}}, f_{\eta_{t}}\right)=\left.\frac{\partial^{2}}{\partial t^{2}}\right|_{t=0} \mathbf{I}\left(f_{\eta_{0}}, f_{\eta_{t}}\right)
$$

(again by fact (3)) and thus the pressure metric is mapping class group invariant.

One may interpret $\mathbf{I}(\rho, \eta)$ as the length in $X_{\eta}$ of a random unit length geodesic on $X_{\rho}$. So, the pressure metric is given by considering the Hessian of the length of a random geodesic. Since the pressure form agrees with Thurston's metric, Wolpert's work [90] implies that the pressure metric is a multiple of the Weil-Petersson metric.

\section{The PRessure metric on the Hitchin COMponent}

Let $V$ be a vector space and $G$ be a group. Recall that a representation $\tau: G \rightarrow \mathrm{GL}(\mathrm{V})$ is irreducible if $\tau(G)$ has no proper invariant subspaces other than $\{0\}$. Let us begin by recalling the following well known result, see for example Humphreys [44].

Proposition 5.1. For each integer $d \geqslant 2$ there exists an irreducible representation $\tau_{d}: \mathrm{SL}_{2}(\mathbb{R}) \rightarrow \mathrm{SL}_{\mathrm{d}}(\mathbb{R})$, unique up to conjugation.

The existence of such an irreducible action is an explicit construction we will now explain. Denote by $\operatorname{Sym}^{d}\left(\mathbb{R}^{2}\right)$ the $d$-dimensional vector space of homogeneous polynomials on 2 variables of degree $d-1$. A base for $\operatorname{Sym}^{d}\left(\mathbb{R}^{2}\right)$ is, for example,

$$
\mathcal{B}=\left\{x^{d-1}, x^{d-2} y, \cdots, x y^{d-2}, y^{d-1}\right\} .
$$

We identify $x$ with $(1,0)$ and $y$ with $(0,1)$ in $\mathbb{R}^{2}$ so that if $g=\left(\begin{array}{ll}a & b \\ c & d\end{array}\right) \in \mathrm{SL}_{2}(\mathbb{R})$, then $g \cdot x=a x+c y$ and $g \cdot y=b x+d y$. The action of $\mathrm{SL}_{2}(\mathbb{R})$ on $\operatorname{Sym}^{d}\left(\mathbb{R}^{2}\right)$ is defined on the base $\mathcal{B}$ by

$$
\tau_{d}(g) \cdot x^{k} y^{d-k-1}=(g \cdot x)^{k}(g \cdot y)^{d-k-1} .
$$

As before, let $S$ be a closed oriented surface of genus $g \geqslant 2$. Hitchin [42] studied the components of the space

$$
\operatorname{Hom}\left(\pi_{1}(S), \mathrm{PSL}_{\mathrm{d}}(\mathbb{R})\right) / \mathrm{PGL}_{\mathrm{d}}(\mathbb{R}) .
$$

containing an element $\rho: \pi_{1}(S) \rightarrow \mathrm{PSL}_{d}(\mathbb{R})$ that factors as

$$
\pi_{1}(S) \stackrel{\rho_{0}}{\longrightarrow} \mathrm{PSL}_{2}(\mathbb{R}) \stackrel{\tau_{d}}{\longrightarrow} \mathrm{PSL}_{\mathrm{d}}(\mathbb{R}),
$$

where $\rho_{0} \in \mathcal{T}(S)$.

By analogy with Teichmüller space, he named these components Teichmüller components, but they are now known as Hitchin components, and denoted by $\mathcal{H}_{d}(S)$. Each Hitchin component contains a copy of $\mathcal{T}(S)$, known 
as the Fuchsian locus, which is an image of $\mathcal{T}(S)$ under the mapping induced by $\tau_{d}$. Hitchin proved the following remarkable result.

Theorem 5.2 (Hitchin [42]). Each Hitchin component $\mathcal{H}_{d}(S)$ is an analytic manifold diffeomorphic to $\mathbb{R}^{\left(d^{2}-1\right)(2 g-2)}=\mathbb{R}^{|\chi(S)| \operatorname{dim} \mathrm{PSL}_{d}(\mathbb{R})}$.

Hitchin [42] commented that "Unfortunately, the analytical point of view used for the proofs gives no indication of the geometrical significance of the Teichmüller component." Labourie [50] introduced dynamical techniques to show that Hitchin representations, i.e. representations in the Hitchin component, are geometrically meaningful. In particular, Hitchin representations are discrete, faithful, quasi-isometric embeddings. Labourie's work significantly expanded the analogy between Hitchin components and Teichmüller spaces.

We view the following result as a further step in exploring this analogy. Its proof follows the same basic strategy as in the Teichmüller space setting, although there are several additional difficulties to overcome.

Theorem 5.3. (Bridgeman-Canary-Labourie-Sambarino [17]) There exists an analytic Riemannian metric on $\mathcal{H}_{d}(S)$ which is invariant under the action of the mapping class group and restricts to a multiple of the Weil-Petersson metric on the Fuchsian locus.

\section{Remark:}

- The mapping class group, regarded as a subgroup of $\operatorname{Out}\left(\pi_{1}(S)\right)$, acts by precomposition on $\mathcal{H}_{d}(S)$.

- When $d=3$ metrics have also been constructed by DarvishzadehGoldman [29] and Qiongling Li [54]. Li [54] showed that both her metric and the metric constructed by Darvishzadeh and Goldman have the properties obtained in our result.

5.1. Labourie's work. Labourie developed the theory of Anosov representations as a tool to study Hitchin representations. This theory was further developed by Guichard and Wienhard [40] and has played a central role in the subsequent development of higher Teichmüller theory. The following theorem summarizes some of the major consequences of Labourie's work for Hitchin representations.

Theorem 5.4. (Labourie $[50,51]$ ) If $\rho \in \mathcal{H}_{d}(S)$ then

(1) $\rho$ is discrete and faithful,

(2) If $\gamma \in \pi_{1}(S)$ is non-trivial, then $\rho(\gamma)$ is diagonalizable over $\mathbb{R}$ with distinct eigenvalues.

(3) $\rho$ is a quasi-isometric embedding.

(4) $\rho$ is irreducible.

Theorem 5.4 is based on Labourie's proof that Hitchin representations are Anosov with respect to a minimal parabolic subgroup for $\operatorname{PSL}_{d}(\mathbb{R})$, i.e. 
the upper triangular matrices in $\mathrm{PSL}_{d}(\mathbb{R})$. We will develop the terminology necessary to give a definition.

A complete flag of $\mathbb{R}^{d}$ is a sequence of vector subspaces $\left\{V_{i}\right\}_{i=1}^{d}$ such that $V_{i} \subset V_{i+1}$ and $\operatorname{dim} V_{i}=i$ for all $i=1, \ldots, d$. Two flags $\left\{V_{i}\right\}$ and $\left\{W_{i}\right\}$ are transverse if $V_{i} \cap W_{d-i}=\{0\}$ for all $i$. Denote by $\mathscr{F}$ the space of complete flags and by $\mathscr{F}^{(2)}$ the space of pairs of transverse flags. The following result should be viewed as the analogue of the limit map constructed in Proposition 4.1 .

Theorem 5.5 (Labourie [50]). If $\rho \in \mathcal{H}_{d}(S)$, then there exists a unique $\rho$-equivariant Hölder map $\xi_{\rho}: \partial_{\infty} \pi_{1}(S) \rightarrow \mathscr{F}$ such that, if $x \neq y$, then the flags $\xi_{\rho}(x)$ and $\xi_{\rho}(y)$ are transverse.

If $\rho \in \mathcal{H}_{d}(S)$ and $x \in \partial_{\infty} \pi_{1}(S)$ then we denote by $\xi_{\rho}^{(k)}(x)$ the $k$-th dimensional space in the flag $\xi_{\rho}(x)$. Notice that if $\gamma_{+}$is an attracting fixed point of the action of $\gamma \in \pi_{1}(S)$ on $\partial_{\infty} \pi_{1}(S)$, then $\xi_{\rho}^{(k)}\left(\gamma_{+}\right)$is spanned by the eigenlines of $\rho(\gamma)$ associated to the $k$ eigenvalues of largest modulus. In particular, $\xi_{\rho}^{(1)}\left(\gamma_{+}\right)$is the attracting fixed point for the action of $\rho(\gamma)$ on $\mathbb{P}\left(\mathbb{R}^{d}\right)$ and $\xi_{\rho}^{(d-1)}\left(\gamma_{-}\right)$is its repelling hyperplane (where $\gamma^{-}$is the repelling fixed point for the action of $\gamma$ on $\left.\pi_{1}(S)\right)$.

When $\rho$ is Fuchsian, Labourie's map is an explicit construction, called the Veronese embedding, which is moreover $\tau_{d}$-equivariant. This is a map from $\partial_{\infty} \mathbb{H}^{2}=\mathbb{P}\left(\mathbb{R}^{2}\right) \rightarrow \mathscr{F}$ explicitly defined, identifying $\mathbb{R}^{d}$ with Sym ${ }^{d}\left(\mathbb{R}^{2}\right)$, by

$$
\mathbb{R} \cdot(a x+b y) \mapsto\left\{p \in \operatorname{Sym}^{d}\left(\mathbb{R}^{2}\right): p \text { has }(a x+b y)^{d-k} \text { as a factor }\right\}_{k=1}^{d} .
$$

Conventions: As in the previous section, we fix $\rho_{0} \in \mathcal{T}(S)$, so that $\rho_{0}$ identifies $S$ with $X_{\rho_{0}}$, and hence identifies $\partial_{\infty} \pi_{1}(S)$ with $\partial_{\infty} \mathbb{H}^{2}$ and $\mathrm{T}^{1} S$ with $\mathrm{T}^{1} X_{\rho_{0}}$. Let $\phi=\phi^{\rho_{0}}$ be the geodesic flow on $S$. Let

$$
\partial_{\infty} \pi_{1}(S)^{(2)}=\left\{(x, y) \in \partial_{\infty} \pi_{1}(S)^{2}: x \neq y\right\}
$$

and consider the Hopf parametrization of $\mathrm{T}^{1} \mathbb{H}^{2}$ by $\partial_{\infty} \pi_{1}(S)^{(2)} \times \mathbb{R}$ where $(x, y, t)$ is the point on the geodesic $L$ joining $x$ to $y$ which is a (signed) distance $t$ from the horocycle through $y$ and a fixed basepoint for $\mathbb{H}^{2}$.

Labourie considers the bundle $E_{\rho}$ over $\mathrm{T}^{1} S$ which is the quotient of $\mathrm{T}^{1} \mathbb{H}^{2} \times \mathscr{F}$ by $\pi_{1}(S)$ where $\gamma \in \pi_{1}(S)$ acts on $\mathrm{T}^{1} \mathbb{H}^{2}$ by $\rho_{0}(\gamma)$ and acts on $\mathscr{F}$ by $\rho(\gamma)$. There is a flow $\tilde{\psi}^{\rho}$ on $\mathrm{T}^{1} \mathbb{H}^{2} \times \mathscr{F}$ which acts by the geodesic flow on $\mathrm{T}^{1} \mathbb{H}^{2}$ and acts trivially on $\mathscr{F}$. The flow $\tilde{\psi}^{\rho}$ descends to a flow $\psi^{\rho}$ on $E_{\rho}$. The limit map $\xi_{\rho}: \partial_{\infty} \pi_{1}(S) \rightarrow \mathscr{F}$ determines a section $\tilde{\sigma}_{\rho}: \mathrm{T}^{1} \mathbb{H}^{2} \rightarrow \tilde{E}_{\rho}$ given by $\tilde{\sigma}(x, y, t)=\left((x, y, t), \xi_{\rho}(x)\right)$ which descends to a section $\sigma: \mathrm{T}^{1} S \rightarrow E_{\rho}$.

A representation $\rho: \pi_{1}(S) \rightarrow \mathrm{PSL}_{\mathrm{d}}(\mathbb{R})$ is Anosov with respect to a minimal parabolic subgroup if and only if there is a limit map with the properties in Theorem 5.5 such that the inverse of the associated flow $\psi^{\rho}$ is contracting on $\sigma_{\rho}\left(\mathrm{T}^{1} S\right)$. 
5.2. The geodesic flow of a Hitchin representation. We wish to associate a topologically transitive metric Anosov flow to each Hitchin representation. Since $\rho$ is discrete and faithful, one is tempted to consider the geodesic flow of the associated locally symmetric space

$$
N_{\rho}=\rho\left(\pi_{1}(S)\right) \backslash \mathrm{PSL}_{\mathrm{d}}(\mathbb{R}) / \mathrm{PSO}(d) .
$$

However, $N_{\rho}$ is neither closed, nor negatively curved, so its geodesic flow will not be Anosov. Moreover, this flow does not even have a nice compact invariant set where it is metric Anosov (see Sambarino [75, Prop. 3.5]).

Sambarino [74, §5] (or more specifically [74, Thm 3.2, Cor. 5.3 and Prop. 5.4]) constructed metric Anosov flows associated to Hitchin representations which are Hölder orbit equivalent to a geodesic flow on a hyperbolic surface such that the closed orbit associated to $\gamma \in \pi_{1}(S)$ has period $\log \Lambda_{\gamma}(\rho)$, where $\Lambda_{\gamma}(\rho)$ is the spectral radius of $\rho(\gamma)$, i.e. the largest modulus of the eigenvalues of $\rho(\gamma)$.

We will use these flows to construct a thermodynamic mapping and an associated pressure metric satisfying the conclusions of Theorem 5.3.

Proposition 5.6 (Sambarino $[74, \S 5]$ ). For every $\rho \in \mathcal{H}_{d}(S)$, there exists a positive Hölder function $f_{\rho}: \mathrm{T}^{1} S \rightarrow(0, \infty)$ such that

$$
\int_{[\gamma]} f_{\rho}=\log \Lambda_{\gamma}(\rho)
$$

for every $\gamma \in \pi_{1}(S)$.

Notice that $\phi^{f_{\rho}}$ is a topologically transitive, metric Anosov flow, Hölder orbit equivalent to the geodesic flow, whose periods are the logarithms of the spectral radii of $\rho\left(\pi_{1}(S)\right)$. We call this flow the geodesic flow of the Hitchin representation.

We will give a different construction of the geodesic flow of a Hitchin representation, from [17], which generalizes easily to the setting of projective Anosov representations of a word-hyperbolic group into $\mathrm{SL}_{d}(\mathbb{R})$. If $\rho \in \mathcal{H}_{d}(S)$, we let $\xi_{\rho}: \partial_{\infty} \pi_{1}(S) \rightarrow \mathscr{F}$ be the associated limit map to the space of complete flags where $\xi_{\rho}(x)=\left\{\xi_{\rho}^{k}(x)\right\}_{k=1}^{d}$. We consider the line bundle $F_{\rho}$ over $\partial_{\infty} \pi_{1}(S)^{(2)}$ whose fiber at $(x, y)$ is $\mathrm{M}(x, y)=$

$\left\{(\varphi, v) \in\left(\mathbb{R}^{d}\right)^{*} \times \mathbb{R}^{d} \mid \operatorname{ker} \varphi=\xi_{\rho}^{(d-1)}(x), v \in \xi_{\rho}^{(1)}(y), \varphi(v)=1\right\} /(\varphi, v) \sim(-\varphi,-v)$.

Consider the flow $\tilde{\phi}^{\rho}=\left(\tilde{\phi}_{t}^{\rho}: F_{\rho} \rightarrow F_{\rho}\right)_{t \in \mathbb{R}}$ given by

$$
\tilde{\phi}_{t}^{\rho}(\varphi, v)=\left(e^{-t} \varphi, e^{t} v\right)
$$

Notice that the $\pi_{1}(S)$ l on $\tilde{F}_{\rho}$ given by

$$
\gamma(x, y,(\varphi, v))=\left(\gamma(x), \gamma(y),\left(\varphi \circ \rho(\gamma)^{-1}, \rho(\gamma) v\right)\right)
$$

is free. We further show that it is properly discontinuous and co-compact, so $\tilde{\phi}^{\rho}$ descends to a flow $\phi^{\rho}$ on $\mathrm{U}_{\rho}=F_{\rho} / \pi_{1}(S)$, which we call the geodesic flow of $\rho$. The proof proceeds by finding a $\rho$-equivariant orbit equivalence between $\mathrm{T}^{1} \mathbb{H}^{2}$ and $F_{\rho}$. 
Proposition 5.7. ([17, Prop. 4.1+Prop 4.2]) The group $\pi_{1}(S)$ acts properly discontinuous and cocompactly on $F_{\rho}$. The quotient flow $\phi^{\rho}$ on $\mathrm{U}_{\rho}$ is Hölder orbit equivalent to the geodesic flow on $\mathrm{T}^{1} S$. Moreover, the closed orbit associated to $\gamma \in \pi_{1}(S)$ has $\phi^{\rho}$-period $\log \Lambda_{\rho}(\gamma)$.

Sketch of proof: Consider the flat bundle $E_{\rho}$ over $\mathrm{T}^{1} S$ which is the quotient of $\mathrm{T}^{1} \mathbb{H}^{2} \times \mathbb{R}^{d}$ by $\pi_{1}(S)$ where $\gamma \in \pi_{1}(S)$ acts on $\mathrm{T}^{1} \mathbb{H}^{2}$ by $\rho_{0}(\gamma)$ and acts on $\mathbb{R}^{d}$ by $\rho(\gamma)$. One considers a flow $\tilde{\psi}^{\rho}$ on $\mathrm{T}^{1} \mathbb{H}^{2} \times \mathbb{R}^{d}$ which acts as the geodesic flow on $\mathrm{T}^{1} \mathbb{H}^{2}$ and acts trivially on $\mathbb{R}^{d}$. The flow $\tilde{\psi}^{\rho}$ preserves the $\rho\left(\pi_{1}(S)\right)$-invariant line sub-bundle $\tilde{\Sigma}$ whose fiber over the point $(x, y, t)$ is $\xi_{\rho}^{(1)}(x)$. Thus, $\tilde{\psi}^{\rho}$ descends to a flow $\psi^{\rho}$ on $E_{\rho}$ preserving the line sub-bundle $\Sigma$ which is the quotient of $\tilde{\Sigma}$. Since $\rho$ is Anosov with respect to a minimal parabolic subgroup, $\psi^{\rho}$ is contracting on $\Sigma$ (see [17, Lem. 2.4]). Since $\psi^{\rho}$ is contracting on $\Sigma$ one may use an averaging procedure to construct a metric $\tau$ on $\Sigma$ with respect to which $\psi^{\rho}$ is uniformly contracting.

Lemma 5.8. ([17, Lemma 4.3]) There exists a Hölder metric $\tau$ on $\Sigma$ and $\beta>0$, so that for all $t>0$,

$$
\left(\psi_{t}^{\rho}\right)_{*}(\tau)<e^{-\beta t} \tau
$$

We construct a $\rho$-equivariant Hölder orbit equivalence

$$
\tilde{j}(x, y, t)=(x, y, u(x, y, t))
$$

where $\tilde{\tau}(u(x, y, t))=1$ and $\tilde{\tau}$ is the lift of $\tau$ to $\tilde{\Sigma}$. The map $\tilde{j}$ is $\rho$-equivariant, since $\xi_{\rho}$ is, and the fact that $\tilde{\tau}$ is uniformly contracting implies that $\tilde{j}$ is injective. It remains to prove that $\tilde{j}$ is proper to show that it is a homeomorphism. (We refer the reader to the proof of Proposition 4.2 in [17] for this relatively simple argument.) Then, $\tilde{j}$ descends to a Hölder orbit equivalence $j$ between $\mathrm{T}^{1} S$ and $U_{\rho}$.

In order to complete the proof, it suffices to evaluate the period of the closed orbit associated to an element $\gamma \in \pi_{1}(S)$. The closed orbit associated to $\gamma$ is the quotient of the fiber of $F_{\rho}$ over $\left(\gamma^{+}, \gamma^{-}\right)$. If we pick $v \in \xi_{\rho}^{(1)}\left(\gamma^{+}\right)$ and $\varphi \in \xi_{\rho}^{(d-1)}\left(\gamma^{-}\right)$so that $\varphi(v)=1$, then

$$
\begin{aligned}
\gamma\left(\gamma^{+}, \gamma^{-},(\varphi, v)\right) & =\left(\gamma^{-}, \gamma^{+},\left( \pm\left(\Lambda_{\rho}(\gamma)\right)^{-1} \varphi, \pm \Lambda_{\rho}(\gamma) v\right)\right) \\
& =\tilde{\phi}_{\log \Lambda_{\rho}(\gamma)}^{\rho}\left(\gamma^{+}, \gamma^{-},(\varphi, v)\right),
\end{aligned}
$$

so the closed orbit has period $\log \Lambda(\rho(\gamma))$ as claimed.

Notice that Proposition 5.6 follows immediately from Proposition 5.7 and Lemma 2.2.

5.3. The thermodynamic mapping. Proposition 5.6 allows us to construct a thermodynamic mapping in the Hitchin setting. Livšic's Theorem (Theorem 2.1) guarantees that if $\rho \in \mathcal{H}_{d}(S)$, then the Livšic cohomology class of the reparametrization function $f_{\rho}$ is well-defined. So, applying 
Lemma 2.3, we may define a thermodynamic mapping

$$
\Phi: \mathcal{H}_{d}(S) \rightarrow \mathcal{H}\left(\mathrm{T}^{1} S\right)
$$

from the Hitchin component $\mathcal{H}_{d}(S)$ to the space $\mathcal{H}\left(\mathrm{T}^{1} S\right)$ of Livšic cohomology classes of pressure zero Hölder functions on $\mathrm{T}^{1} S$, by letting

$$
\Phi(\rho)=\left[-h(\rho) f_{\rho}\right] .
$$

In order to construct an analytic pressure form, we need to know that $\Phi$ admits local analytic lifts to the space $\mathcal{P}\left(\mathrm{T}^{1} S\right)$ of pressure zero Hölder functions on $\mathrm{T}^{1} S$.

Proposition 5.9 ([17, Prop. 6.2]). The mapping $\Phi$ admits local analytic lifts to the space $\mathcal{P}\left(\mathrm{T}^{1} S\right)$, i.e. each $\rho \in \mathcal{H}_{d}(S)$ has an open neighborhood $W$ and an analytic map $\tilde{\Phi}: W \rightarrow \mathcal{P}\left(\mathrm{T}^{1} S\right)$ such that $\Phi(\rho)=[\tilde{\Phi}(\rho)]$.

Sketch of proof: Let $\rho \in \mathcal{H}_{d}(S)$. Choose a neighborhood $V$ of $\rho$ which we may implicitly identify with a submanifold of $\operatorname{Hom}\left(\pi_{1}(S), \mathrm{PSL}_{\mathrm{d}}(\mathbb{R})\right.$ ) (by an analytic map whose composition with the projection map is the identity). Consider the $\mathscr{F}$-bundle $\tilde{A}=V \times \mathrm{T}^{1} \mathbb{H}^{2} \times \mathscr{F}$ over $V \times \mathrm{T}^{1} \mathbb{H}^{2}$. There is a natural action of $\pi_{1}(S)$ on $\tilde{A}$ so that $\gamma \in \pi_{1}(S)$ take $(\eta,(x, y, t), F)$ to $(\eta, \gamma(x, y, t), \eta(\gamma) F))$ with quotient $A$. The limit map $\xi_{\rho}$ determines a section $\sigma_{\rho}$ of $A$ over $\{\rho\} \times \mathrm{T}^{1} S$.

The geodesic flow on $\mathrm{T}^{1} S$ lifts to a flow $\left\{\Psi_{t}\right\}_{t \in \mathbb{R}}$ on $A$ (whose lift to $\tilde{A}$ acts trivially in the $V$ and $\mathscr{F}$ direction). The Anosov property of Hitchin representations implies that the inverse flow is contracting on $\sigma_{\rho}\left(\{\rho\} \times \mathrm{T}^{1} S\right)$. One may extend $\sigma_{\rho}$ to a section $\sigma$ of $A$ over $V \times \mathrm{T}^{1} S$ which varies analytically in the $V$ coordinate (after first possibly restricting to a smaller neighborhood of the lift of $\rho$ ). One may now apply the machinery developed by HirschPugh-Shub [41] (see also Shub [78]), to find a section $\tau$ of $A$ over $W \times \mathrm{T}^{1} S$, where $W$ is a sub-neighborhood of $V$, so that the inverse flow preserves and is contracting along $\tau\left(W \times \mathrm{T}^{1} S\right)$. Here the main idea is to apply the contraction mapping theorem cleverly to show that one may take

$$
\tau(\eta, X)=\lim \Psi_{-n t_{0}}\left(\sigma\left(\eta, \Psi_{n t_{0}}(x)\right)\right)
$$

for some $t_{0}>0$ so that $\Psi_{-t_{0}}$ is uniformly contracting. It follows from standard techniques that $\tau$ varies smoothly in the $W$ direction and that the restriction to $\{\eta\} \times \mathrm{T}^{1} S$ is Hölder for all $\eta \in W$. One must complexify the situation by considering representations into $\mathrm{PSL}_{d}(\mathbb{C})$ in order to verify that $\tau$ varies analytically in the $W$ direction. (See Section 6 of [17] for more details).

The section $\tau$ lifts to a section $\tilde{\tau}$ of $\tilde{A}$ which is induced by a map

$$
\hat{\xi}: W \times \partial_{\infty} \pi_{1}(S) \rightarrow \mathscr{F}
$$

which varies analytically in the $W$ direction such that

$$
\hat{\xi}_{\eta}=\hat{\xi}(\eta, \cdot): \partial_{\infty} \pi_{1}(S) \rightarrow \mathbb{P}\left(\mathbb{R}^{d}\right)
$$


is $\eta$-equivariant and Hölder for all $\eta \in W$. The uniqueness of limit maps for Hitchin representations guarantees that $\hat{\xi}_{\eta}=\xi_{\eta}$. So, $\xi_{\eta}$ varies analytically over $W$.

One may then examine the proof of Proposition 5.7 and apply an averaging procedure, as in the Teichmüller space case, to produce an analytically varying family of Hölder function $\left\{f_{\eta}\right\}_{\eta \in W}$, so that the reparametrization of the geodesic flow on $\mathrm{T}^{1} S$ by $f_{\eta}$ has the same periods as $U_{\eta}$. (Again to get analytic, rather than just smooth, variation one must complexify the situation. See Section 6 of [17] for details.) Therefore, the map

$$
\tilde{\Phi}: W \rightarrow \mathcal{P}\left(\mathrm{T}^{1} S\right)
$$

given by

$$
\tilde{\Phi}(\eta)=-h(\eta) f_{\eta}
$$

is an analytic local lift of $\Phi$.

5.4. Entropy and intersection numbers. Proposition 5.6 allows us to define entropy and intersection numbers for Hitchin representations. If $\rho \in \mathcal{H}_{d}(S)$, let

$$
R_{T}(\rho)=\left\{[\gamma] \in\left[\pi_{1}(S)\right] \mid \log \left(\Lambda_{\rho}(\gamma)\right) \leq T\right\} .
$$

The entropy of $\rho$ is given by

$$
h(\rho)=h\left(f_{\rho}\right)=\lim _{T \rightarrow \infty} \frac{\log \# R_{T}(\rho)}{T} .
$$

The intersection number of $\rho$ and $\eta$ in $\mathcal{H}_{d}(S)$ is given by

$$
\mathbf{I}(\rho, \eta)=\mathbf{I}\left(f_{\rho}, f_{\eta}\right)=\lim _{T \rightarrow \infty} \frac{1}{\# R_{T}(\rho)} \sum_{[\gamma] \in R_{\rho}(T)} \frac{\log \left(\Lambda_{\eta}(\gamma)\right)}{\log \left(\Lambda_{\rho}(\gamma)\right)}
$$

and their renormalized intersection number is

$$
\mathbf{J}(\rho, \eta)=\mathbf{J}\left(f_{\rho}, f_{\eta}\right)=\frac{h(\eta)}{h(\rho)} \mathbf{I}(\rho, \eta) .
$$

Proposition 5.9 and Corollary 2.6 immediately give:

Corollary 5.10. Entropy varies analytically over $\mathcal{H}_{d}(S)$ and intersection $\mathbf{I}$ and renormalized intersection $\mathbf{J}$ vary analytically over $\mathcal{H}_{d}(S) \times \mathcal{H}_{d}(S)$.

Remark: It follows from Bonahon's work [8], that the intersection number is symmetric on Teichmüller space. However, it is clear that the intersection number is not symmetric on the Hitchin component. For example, one may use the work of Zhang [94, 95] to exhibit for all $K>1$ and $d \geq 3, \rho_{1}, \rho_{2} \in$ $\mathcal{H}_{d}(S)$ such that $\log \Lambda\left(\rho_{1}(\gamma)\right) \geq K \log \Lambda\left(\rho_{2}(\gamma)\right)$ for all $\gamma \in \pi_{1}(S)-\{i d\}$, so $\mathbf{I}\left(\rho_{1}, \rho_{2}\right) \geq K$ and $\mathbf{I}\left(\rho_{2}, \rho_{1}\right) \leq \frac{1}{K}$. One expects that the renormalized intersection number is also asymmetric. 
5.5. The pressure form. We then define the analytic pressure form on $\mathcal{H}_{d}(S)$ as the pullback of the pressure form on $\mathcal{P}\left(\mathrm{T}^{1} S\right)$ using a lift of the thermodynamic mapping $\Phi$. Explicitly, if $\left\{\eta_{t}\right\}_{t \in(-1,1)}$ is an analytic path in $\mathcal{H}_{d}(S)$ and $\tilde{\Phi}: U \rightarrow \mathcal{P}\left(\mathrm{T}^{1} S\right)$ is an analytic lift of $\Phi$ defined on a neighborhood $U$ of $\rho$, then we define

$$
\left\|\dot{\eta}_{0}\right\|_{\mathbf{P}}^{2}=\left\|d \tilde{\Phi}\left(\dot{\eta}_{0}\right)\right\|_{\mathbf{P}}^{2}
$$

If $\rho \in \mathcal{H}_{d}(S)$ and $\mathbf{J}_{\rho}: \mathcal{H}_{d}(S) \rightarrow \mathbb{R}$ is defined by

$$
\mathbf{J}_{\rho}(\eta)=\mathbf{J}(\rho, \eta)=\mathbf{J}\left(f_{\rho}, f_{\eta}\right),
$$

then Proposition 2.8 implies that the pressure form on $\mathrm{T}_{\rho} \mathcal{H}_{d}(S)$ is the Hessian of $\mathbf{J}_{\rho}$ at $\rho$. Since the renormalized intersection number is mapping class group invariant by definition, it follows that the pressure form is also mapping class group invariant. Wolpert's theorem [90] implies that the restriction of the pressure form to the Fuchsian locus is a multiple of the WeilPetersson metric. It only remains to show that the pressure form is positive definite, so gives rise to an analytic Riemannian metric on all of $\mathcal{H}_{d}(S)$.

5.6. Non-degeneracy of the pressure metric. We complete the proof of Theorem 5.3 by proving:

Proposition 5.11. The pressure form is non-degenerate at each point in $\mathcal{H}_{d}(S)$.

We note that each Hitchin component $\mathcal{H}_{d}(S)$ lifts to a component of

$$
X\left(\pi_{1}(S), \mathrm{SL}_{d}(\mathbb{R})\right)=\operatorname{Hom}\left(\pi_{1}(S), \mathrm{SL}_{d}(\mathbb{R})\right) / \mathrm{SL}_{d}(\mathbb{R})
$$

and we will work in this lift throughout the proof (see Hitchin [42, Section 10].)

In particular, this allows us to define, for all $\gamma \in \pi_{1}(S)$, an analytic function $\operatorname{Tr}_{\gamma}: \mathcal{H}_{d}(S) \rightarrow \mathbb{R}$, where $\operatorname{Tr}_{\gamma}(\rho)$ is the trace of $\rho(\gamma)$.

As in the Teichmüller case, the proof proceeds by applying Corollary 2.5. If $\left\{\eta_{t}\right\}_{(-\varepsilon, \varepsilon)} \subset \mathcal{H}_{d}(S)$ is a path such that $\left\|\dot{\eta}_{0}\right\|_{\mathbf{P}}=\|d \Phi v\|_{\mathbf{P}}=0$, then

$$
\left.\frac{\partial}{\partial t}\right|_{t=0} h\left(f_{\eta_{t}}\right) \ell_{f_{\eta_{t}}}(\gamma)=0
$$

for all $\gamma \in \pi_{1}(S)$. The main difference is that entropy is not constant in the Hitchin component.

If $\gamma \in \pi_{1}(S)$, we may think of $\log \Lambda_{\gamma}$ as an analytic function on $\mathcal{H}_{d}(S)$, where we recall that $\Lambda_{\gamma}(\rho)$ is the spectral radius of $\rho(\gamma)$. The following lemma is an immediate consequence of Equation (4) (compare with Equation (2) in Section 4.4).

Lemma 5.12. If $v \in \mathrm{T}_{\rho} \mathcal{H}_{d}(S)$ and $\left\|D_{\rho} \Phi(v)\right\|_{\mathbf{P}}=0$, then

$$
D_{\rho} \log \Lambda_{\gamma}(v)=-\frac{D_{\rho} h(v)}{h(\rho)} \log \Lambda_{\gamma}(\rho)
$$

for all $\gamma \in \pi_{1}(S)$. 
Lemma 5.12 implies that if $v \in \mathrm{T}_{\rho} \mathcal{H}_{d}(S)$ is a degenerate vector, and we set $K=-\frac{D_{\rho} h(v)}{h(\rho)}$, then $D_{\rho} \log \Lambda_{\gamma}(v)=K \log \Lambda_{\gamma}(\rho)$ for all $\gamma \in \pi_{1}(S)$. The next proposition, which is the key step in the proof of Proposition 5.11, then guarantees that the derivative of the trace function of every element is trivial in the direction $v$.

Proposition 5.13. If $v \in \mathrm{T}_{\rho} \mathcal{H}_{d}(S)$ and there exists $K \in \mathbb{R}$ such that

$$
D_{\rho} \log \Lambda_{\gamma}(v)=K \log \Lambda_{\gamma}(\rho)
$$

for all $\gamma \in \pi_{1}(S)$, then $K=0$ and $D_{\rho} \operatorname{Tr}_{\gamma}(v)=0$ for all $\gamma \in \pi_{1}(S)$.

The proof of Proposition 5.11, and hence Theorem 5.3, is then completed by applying the following standard lemma.

Lemma 5.14. If $\rho \in \mathcal{H}_{d}(S)$, then $\left\{D_{\rho} \operatorname{Tr}_{\gamma} \mid \gamma \in \pi_{1}(S)\right\}$ spans the cotangent space $\mathrm{T}_{\rho}^{*} \mathcal{H}_{d}(S)$.

Since every Hitchin representation is absolutely irreducible, Schur's Lemma can be used to show that $\mathcal{H}_{d}(S)$ immerses in the $\mathrm{SL}_{d}(\mathbb{C})$-character variety $X\left(\pi_{1}(S), \mathrm{SL}_{d}(\mathbb{C})\right)$. Lemma 5.14 then follows from standard facts about $X\left(\pi_{1}(S), \mathrm{SL}_{d}(\mathbb{C})\right)$ (see Lubotzky-Magid [57]).

Proof of Proposition 5.13: It will be useful to introduce some notation. If $M$ is a real analytic manifold, an analytic function $f: M \rightarrow \mathbb{R}$ has log-type $K$ at $v \in \mathrm{T}_{u} M$ if $f(u) \neq 0$ and

$$
\mathrm{D}_{u} \log (|f|)(v)=K \log (|f(u)|) .
$$

Suppose that $A \in \mathrm{SL}_{d}(\mathbb{R})$ has real eigenvalues $\left\{\lambda_{i}(A)\right\}_{i=1}^{n}$ where

$$
\left|\lambda_{1}(A)\right|>\left|\lambda_{2}(A)\right|>\ldots>\left|\lambda_{m}(A)\right| \text {. }
$$

If $\mathbf{p}_{i}(A)$ is the projection onto the $\lambda_{i}(A)$-eigenspace parallel to the hyperplane spanned by the other eigenspaces, then

$$
A=\sum_{k=1}^{m} \lambda_{k}(A) \mathbf{p}_{i}(A) .
$$

We say that two infinite order elements of $\pi_{1}(S)$ are coprime if they do not share a common power. The following lemma is an elementary computation (see Benoist [4, Cor 1.6] or [17, Prop. 9.4]).

Lemma 5.15. If $\alpha$ and $\beta$ are coprime elements of $\pi_{1}(S)$ and $\rho \in \mathcal{H}_{d}(S)$, then

$$
\operatorname{Tr}\left(\mathbf{p}_{1}(\rho(\alpha)) \mathbf{p}_{1}(\rho(\beta))\right)=\lim _{n \rightarrow \infty} \frac{\lambda_{1}\left(\rho\left(\alpha^{n} \beta^{n}\right)\right)}{\lambda_{1}\left(\rho\left(\alpha^{n}\right)\right) \lambda_{1}\left(\rho\left(\beta^{n}\right)\right)} \neq 0
$$

and

$$
\operatorname{Tr}\left(\mathbf{p}_{1}(\rho(\alpha)) \rho(\beta)\right)=\lim _{n \rightarrow \infty} \frac{\lambda_{1}\left(\rho\left(\alpha^{n} \beta\right)\right)}{\lambda_{1}\left(\rho\left(\alpha^{n}\right)\right)} \neq 0
$$

for all $\rho \in \mathcal{H}_{d}(S)$. 
The following rather technical lemma plays a key role in the proof of Proposition 5.13.

Lemma 5.16. Suppose that $\left\{a_{p}\right\}_{p=1}^{q},\left\{u_{p}\right\}_{p=1}^{q},\left\{b_{s}\right\}_{s=1}^{\infty}$, and $\left\{v_{s}\right\}_{s=1}^{\infty}$ are collections of real numbers so that $\left\{\left|u_{p}\right|\right\}_{p=1}^{q}$ and $\left\{\left|v_{s}\right|\right\}_{s=1}^{\infty}$ are strictly decreasing, each $u_{p}$ is non-zero,

$$
\sum_{p=1}^{q} n a_{p} u_{p}^{n}=\sum_{s=1}^{\infty} b_{s} v_{s}^{n}
$$

for all $n>0$, and $\sum_{s=1}^{\infty} b_{s} v_{s}$ is absolutely convergent. Then, $a_{p}=0$ for all $p$.

Proof. We may assume without loss of generality that each $b_{s}$ is non-zero. We divide each side of the equality by $n u_{1}^{n}$, to see that

$$
a_{1}=\lim _{n \rightarrow \infty} \sum_{s=1}^{\infty}\left(\frac{b_{s}}{n}\right) \frac{v_{s}^{n}}{u_{1}^{n}}
$$

for all $n$. However, the right hand side of the equation can only be bounded as $n \rightarrow \infty$, if $\left|v_{1}\right| \leq\left|u_{1}\right|$. However, if $\left|v_{1}\right| \leq\left|u_{1}\right|$, then the limit of the right hand side, as $n \rightarrow \infty$, must be 0 and we conclude that $a_{1}=0$.

We may iterate this procedure to conclude that $a_{p}=0$ for all $p$.

Suppose that $\alpha, \beta \in \pi_{1}(S)$ are coprime. We consider the analytic function $F_{n}: \mathcal{H}_{d}(S) \rightarrow \mathbb{R}$ given by

$$
F_{n}(\rho)=\frac{\operatorname{Tr}\left(\mathbf{p}_{1}(\rho(\alpha)) \rho\left(\beta^{n}\right)\right)}{\lambda_{1}\left(\rho\left(\beta^{n}\right)\right) \operatorname{Tr}\left(\mathbf{p}_{1}(\rho(\alpha)) \mathbf{p}_{1}(\rho(\beta))\right)} .
$$

Lemma 5.15 and the assumption of Proposition 5.13 imply that $F_{n}$ is of log-type $K$ at $v$ (see the proofs of Proposition 9.4 and Lemma 9.8 in [17]). Using equation (5) we have

$$
\rho\left(\beta^{n}\right)=\sum_{k=1}^{d} \lambda_{k}(\rho(\beta))^{n} \mathbf{p}_{k}(\rho(\beta)) .
$$

Thus, we can write $F_{n}$ as

$$
F_{n}(\rho)=1+\sum_{k=2}^{d} \frac{\operatorname{Tr}\left(\mathbf{p}_{1}(\rho(\alpha)) \mathbf{p}_{k}(\rho(\beta))\right)}{\operatorname{Tr}\left(\mathbf{p}_{1}(\rho(\alpha)) \mathbf{p}_{1}(\rho(\beta))\right)}\left(\frac{\lambda_{k}(\rho(\beta))}{\lambda_{1}(\rho(\beta))}\right)^{n}=1+\sum_{k=2}^{d} f_{k} t_{k}^{n}
$$

where

$f_{k}(\rho)=\frac{\operatorname{Tr}\left(\mathbf{p}_{1}(\rho(\alpha)) \mathbf{p}_{k}(\rho(\beta))\right)}{\operatorname{Tr}\left(\mathbf{p}_{1}(\rho(\alpha)) \mathbf{p}_{1}(\rho(\beta))\right)} \neq 0 \quad$ and $\quad t_{k}(\rho)=\left(\frac{\lambda_{k}(\rho(\beta))}{\left|\lambda_{1}(\rho(\beta))\right|}\right) \neq 0$. 
Since $F_{n}$ has log-type $K$ at $v$ and is positive in some neighborhood of $\rho$,

$$
D_{\rho} F_{n}(v)=\sum_{k=2}^{d} n f_{k} t_{k}^{n} \frac{\dot{t}_{k}}{t_{k}}+\sum_{k=2}^{d} \dot{f}_{k} t_{k}^{n}=K F_{n}(\rho) \log \left(F_{n}(\rho)\right)
$$

where $\dot{t}_{k}=D_{\rho} t_{k}(v)$ and $\dot{f}_{k}=D_{\rho} a_{k}(v)$. In order to simplify the proof, we consider Equation (6) for even powers. Using the Taylor series expansion for $\log (1+x)$ and grouping terms we have

$$
F_{2 n} \log \left(F_{2 n}\right)=\left(1+\sum_{k=2}^{d} f_{k} t_{k}^{2 n}\right) \log \left(1+\sum_{k=2}^{d} f_{k} t_{k}^{2 n}\right)=\sum_{s=1}^{\infty} c_{s} w_{s}^{n}
$$

where $\left\{w_{s}\right\}$ is a strictly decreasing sequence of positive terms. We may again regroup terms to obtain

$$
\sum_{k=2}^{d} 2 n\left(\frac{f_{k} \dot{t}_{k}}{t_{k}}\right) t_{k}^{2 n}=\sum_{s=1}^{\infty} c_{s} w_{s}^{n}-\sum_{k=2}^{d} \dot{f}_{k} t_{k}^{2 n}=\sum_{s=1}^{\infty} b_{s} v_{s}^{n}
$$

where $\left\{v_{s}\right\}$ is a strictly decreasing sequence of positive terms. So, letting $u_{k}=t_{k}^{2}$, we see that for all $n$

$$
\sum_{k=2}^{d} n\left(\frac{2 f_{k} \dot{t}_{k}}{t_{k}}\right) u_{k}^{n}=\sum_{s=1}^{\infty} b_{s} v_{s}^{n}
$$

Lemma 5.16 implies that $\frac{f_{k} \dot{t}_{k}}{t_{k}}=0$ for all $k$, so $\dot{t}_{k}=0$ for all $k$. Let $\lambda_{i, \beta}$ be the real-valued analytic function on $\mathcal{H}_{d}(S)$ given by $\lambda_{i, \beta}(\rho)=\lambda_{i}(\rho(\beta))$. Then,

$$
\frac{\dot{\lambda}_{k, \beta} \lambda_{1, \beta}-\dot{\lambda}_{1, \beta} \lambda_{k, \beta}}{\lambda_{1, \beta}^{2}}=0
$$

So,

$$
D_{\rho}\left(\log \left(\left|\lambda_{k, \beta}\right|\right)\right)(v)=\frac{\dot{\lambda}_{k, \beta}}{\lambda_{k, \beta}}=\frac{\dot{\lambda}_{1, \beta}}{\lambda_{1, \beta}}=D_{\rho}\left(\log \left(\left|\lambda_{1, \beta}\right|\right)\right)(v)=K \log \left(\left|\lambda_{1, \beta}(\rho)\right|\right) .
$$

Since $\lambda_{d, \beta}=\frac{1}{\lambda_{1, \beta^{-1}}}$,

$$
\begin{aligned}
K \log \left(\left|\lambda_{1, \beta^{-1}}(\rho)\right|\right) & =D_{\rho}\left(\log \left(\left|\lambda_{1, \beta^{-1}}\right|\right)\right)(v)=D_{\rho}\left(\log \left(\left|\lambda_{d, \beta^{-1}}\right|\right)\right)(v) \\
& =-D_{\rho}\left(\log \left(\left|\lambda_{1, \beta}\right|\right)\right)(v)=-K \log \left(\left|\lambda_{1, \beta}(\rho)\right|\right) .
\end{aligned}
$$

Therefore, since $\log \left(\left|\lambda_{1, \beta^{-1}}(\rho)\right|\right)$ and $\log \left(\left|\lambda_{1, \beta}(\rho)\right|\right)$ are both positive, $K=0$, which implies that $\dot{\lambda}_{k}(\beta)=0$ for all $k$.

Since, $D_{\rho} \lambda_{i, \beta}(v)=0$ for all $i$ and all $\beta, D_{\rho} \operatorname{Tr}_{\beta}=0$ for all $\beta \in \pi_{1}(S)$. This completes the proof of Proposition 5.13, and hence Proposition 5.11 and Theorem 5.3.

\subsection{Rigidity theorems for Hitchin representations.}


5.7.1. Entropy rigidity. Potrie and Sambarino recently showed that entropy is maximized only along the Fuchsian locus. One may view this as an analogue of Bowen's celebrated result [10] that the topological entropy of a quasifuchsian group is at least 1 and it is 1 if and only if the group is Fuchsian.

Theorem 5.17. (Potrie-Sambarino [71]) If $\rho \in \mathcal{H}_{d}(S)$, then $h(\rho) \leq \frac{2}{d-1}$. Moreover, if $h(\rho)=\frac{2}{d-1}$, then $\rho$ lies in the Fuchsian locus.

Remarks: (1) Crampon [27] had earlier established that the entropy associated to Hilbert length (see Section 5.8) of holonomies of strictly convex projective structures on closed hyperbolic manifolds is maximal exactly at the representations into $\mathrm{SO}(d, 1)$. In particular, the Hilbert length entropy on $\mathcal{H}_{3}(S)$ is maximal exactly at the Fuchsian locus.

(2) Tengren Zhang $[94,95]$ showed that, for all $d$, there exist large families of sequences of Hitchin representations with entropy converging to 0 . Nie [64] had earlier constructed specific examples when $d=3$.

5.7.2. Intersection number and marked length rigidity theorems. One also obtains the following rigidity theorem for Hitchin representations with respect to the intersection number. Notice that the definition of the renormalized intersection number $\mathbf{J}\left(\rho_{1}, \rho_{2}\right)$ for $\rho_{1} \in \mathcal{H}_{d_{1}}(S)$ and $\rho_{2} \in \mathcal{H}_{d_{2}}(S)$ makes sense even if $d_{1} \neq d_{2}$, see Section 5.4. Moreover, if $f_{1}: \mathrm{T}^{1} S \rightarrow \mathbb{R}$ and $f_{2}: \mathrm{T}^{1} S \rightarrow \mathbb{R}$ are positive Hölder functions such that $\phi^{f_{1}}=U_{\rho_{1}}$ and $\phi^{f_{2}}=$ $U_{\rho_{2}}$, then $\mathbf{J}\left(\rho_{1}, \rho_{2}\right)=\mathbf{J}\left(f_{1}, f_{2}\right)$. In particular, see Lemma 2.7, $\mathbf{J}\left(\rho_{1}, \rho_{2}\right) \geq 1$.

Theorem 5.18. ([17, Cor. 1.5]) Let $S$ be a closed, orientable surface and let $\rho_{1} \in \mathcal{H}_{d_{1}}(S)$ and $\rho_{2} \in \mathcal{H}_{d_{2}}(S)$ be two Hitchin representations such that

$$
\mathbf{J}\left(\rho_{1}, \rho_{2}\right)=1 \text {. }
$$

Then, either

(1) $d_{1}=d_{2}$ and $\rho_{1}=\rho_{2}$ in $\mathcal{H}_{d_{1}}(S)$, or

(2) there exists an element $\rho$ of the Teichmüller space $\mathcal{T}(S)$ so that $\rho_{1}=$ $\tau_{d_{1}}(\rho)$ and $\rho_{2}=\tau_{d_{2}}(\rho)$.

The proof of Theorem 5.18 makes use of general rigidity results in the Thermodynamic Formalism and a result of Guichard [39] classifying Zariski closures of images of Hitchin representations.

As an immediate corollary, one obtains a marked length rigidity theorem where one uses the logarithm of the spectral radius as a notion of length.

Corollary 5.19. If $\rho_{1}, \rho_{2} \in \mathcal{H}_{d}(S)$, then

$$
\frac{h\left(\rho_{1}\right)}{h\left(\rho_{2}\right)} \sup _{\gamma \in \pi_{1}(S)} \frac{\Lambda_{\gamma}\left(\rho_{1}\right)}{\Lambda_{\gamma}\left(\rho_{2}\right)} \geqslant 1
$$

with equality if and only if there exists $g \in \mathrm{GL}_{d}(\mathbb{R})$ such that $g \rho_{1} g^{-1}=\rho_{2}$. In particular, if $\Lambda_{\gamma}\left(\rho_{1}\right)=\Lambda_{\gamma}\left(\rho_{2}\right)$ for all $\gamma \in \pi_{1}(S)$, then $\rho_{1}$ and $\rho_{2}$ are conjugate in $\mathrm{GL}_{d}(\mathbb{R})$. 
Bridgeman, Canary and Labourie have recently established that it suffices to consider lengths of simple closed curves.

Theorem 5.20. (Bridgeman-Canary-Labourie [16]) If $\rho_{1}, \rho_{2} \in \mathcal{H}_{d}(S)$ and $\Lambda_{\alpha}\left(\rho_{1}\right)=\Lambda_{\alpha}\left(\rho_{2}\right)$ for all $\alpha \in \pi_{1}(S)$ which are represented by simple closed curves, then $\rho_{1}$ and $\rho_{2}$ are conjugate in $\mathrm{PGL}_{d}(\mathbb{R})$.

Remarks: Burger [19] introduced a renormalized intersection number between convex cocompact representations into rank one Lie groups and proved an analogue of Theorem 5.18 in that setting. One should compare Corollary 5.19 and Theorem 5.20 with the marked length spectrum rigidity theorem of Dal'bo-Kim [28] for Zariski dense representations. Both Dal'bo-Kim [28] and Theorem 5.18 rely crucially on work of Benoist [3, Thm. 1.2]. However, the proof of Theorem 5.20 uses Labourie's equivariant Frenet map into the flag variety, see Theorem 5.5, and the theory of positive representations developed by Fock and Goncharov [31].

5.8. An alternate length function. Throughout the section, we have used the logarithm of the spectral radius as a notion of length. It is also quite natural to consider the length of $\rho(\gamma)$ to be

$$
\ell_{\mathbf{H}}(\rho(\gamma))=\log \Lambda(\rho(\gamma))+\log \Lambda\left(\rho\left(\gamma^{-1}\right)\right) .
$$

For example, if $\rho \in \mathcal{H}_{3}(S)$, then $\rho$ is the holonomy of a convex projective structure on $S$, and $\ell_{\mathbf{H}}(\rho(\gamma))$ is the translation length of $\gamma$ in the associated Hilbert metric on $S$.

Sambarino [74] also proves that there is a reparametrization of $\mathrm{T}^{1} S$ whose periods are given by $\ell_{\mathbf{H}}(\rho(\gamma))$.

Proposition 5.21. (Sambarino [74, Thm. 3.2, §5]) If $\rho \in \mathcal{H}_{d}(S)$, then there exists a positive Hölder function $f_{\rho}^{\mathrm{H}}: \mathrm{T}^{1} S \rightarrow(0, \infty)$ such that

$$
\int_{[\gamma]} f_{\rho}^{\mathbf{H}}=\ell_{\mathbf{H}}(\rho(\gamma))
$$

for all $\gamma \in \pi_{1}(S)$.

We give a proof which uses a cross ratio to construct $f_{\rho}^{\mathrm{H}}$ from the limit map $\xi_{\rho}$, as is done in the Teichmüller setting. It is adapted from the construction given in section 3 of Labourie [51].

Proof. Given linear forms $\varphi, \psi \in\left(\mathbb{R}^{d}\right)^{*}$ and vectors $v, w \in \mathbb{R}^{d}$ such that $v \notin \operatorname{ker} \psi$ and $w \notin \operatorname{ker} \varphi$, define the cross-ratio

$$
[\varphi, \psi, v, w]=\frac{\varphi(v) \psi(w)}{\varphi(w) \psi(v)} .
$$

Note that the cross ratio only depends on the projective classes of $\varphi, \psi$, $v$, and $w$, and is invariant under $\mathrm{PSL}_{d}(\mathbb{R})$. Moreover, if $g \in \mathrm{PSL}_{\mathrm{d}}(\mathbb{R})$ is bi-proximal and $v \notin \operatorname{ker} g_{-} \cup \operatorname{ker}\left(g^{-1}\right)_{-}$, then

$$
\left[g_{-},\left(g^{-1}\right)_{-}, v, g v\right]=\Lambda(g) \Lambda\left(g^{-1}\right)
$$


where $g_{-}$is a linear functional whose kernel is the repelling hyperplane of $g$.

Theorem 5.5 provides a $\rho$-equivariant map $\xi_{\rho}: \partial_{\infty} \mathbb{H}^{2} \rightarrow \mathscr{F}$. Define $\kappa_{\eta}: \mathrm{T}^{1} \mathbb{H}^{2} \times \mathbb{R} \rightarrow \mathbb{R}$ by

$$
\kappa_{\eta}((x, y, z), t)=\log \left|\left[\xi_{\eta}^{(d-1)}(x), \xi_{\eta}^{(d-1)}(z), \xi_{\eta}^{(1)}(y), \xi_{\eta}^{(1)}\left(u_{t}(x, y, z)\right)\right]\right|
$$

where $u_{t}$ is determined by $\phi_{t}(x, y, z)=\left(x, u_{t}(x, y, z), z\right)$. Work of Labourie [51, §3] implies that $t \mapsto \kappa_{\eta}((x, y, z), t)$ is an increasing homeomorphism of $\mathbb{R}$, so averaging $\kappa_{\eta}$ and taking derivatives as before provides the desired function $f_{\eta}^{\mathrm{H}}: \mathrm{T}^{1} S \rightarrow(0, \infty)$. Equation (7) implies that $f_{\eta}^{\mathrm{H}}$ has the desired periods.

We may again obtain a thermodynamic mapping $\Phi^{\mathrm{H}}: \mathcal{H}_{d}(S) \rightarrow \mathcal{H}\left(\mathrm{T}^{1} S\right)$ defined by

$$
\eta \mapsto\left[-h\left(f_{\eta}^{\mathrm{H}}\right) f_{\eta}^{\mathrm{H}}\right]
$$

One can use the same arguments as above to show that $\Phi^{\mathrm{H}}$ has locally analytic lifts and one can pull-back the pressure form via $\Phi^{\mathrm{H}}$ to obtain an analytic pressure semi-norm $\|\cdot\|_{\mathrm{H}}$ on $\mathrm{TH}_{d}(S)$. (Pollicott and Sharp [69] previously proved that the entropy associated to $\ell_{\mathrm{H}}$ varies analytically over $\mathcal{H}_{d}(S)$.) However, this pressure form is degenerate in ways which are completely analogous to the degeneracy of the pressure metric on quasifuchsian space discovered by Bridgeman [14].

Consider the contragredient involution $\sigma: \mathrm{PSL}_{d}(\mathbb{R}) \rightarrow \mathrm{PSL}_{d}(\mathbb{R})$ given by $g \mapsto\left(g^{-1}\right)^{\mathrm{t}}$, where $\mathrm{t}$ denotes the transpose operator associated to the standard inner product of $\mathbb{R}^{d}$. This involution induces an involution on the Hitchin component $\hat{\sigma}: \mathcal{H}_{d}(S) \rightarrow \mathcal{H}_{d}(S)$, where $\hat{\sigma}(\rho)(\gamma)=\sigma(\rho(\gamma))$ for all $\gamma \in \pi_{1}(S)$. If $\eta \in \mathcal{H}_{d}(S)$ is a representation whose image lies in (a group conjugate to) $\mathrm{Sp}(2 n, \mathbb{R})$ (if $d=2 n)$ or $\mathrm{SO}(n, n+1, \mathbb{R})($ if $d=2 n+1)$, then $\hat{\sigma}(\eta)=\eta$.

Consider the tangent vectors in $\mathrm{TH}_{d}(S)$ which are reversed by $D \hat{\sigma}$, i.e. let

$$
\mathrm{B}_{\mathrm{d}}(\mathrm{S})=\left\{v \in \mathrm{TH}_{d}(S): D \hat{\sigma}(v)=-v\right\} .
$$

The vectors in $B_{d}(S)$ are degenerate for the pressure metric $\|\cdot\|_{H}$.

Lemma 5.22. If $v \in \mathrm{B}_{\mathrm{d}}(\mathrm{S})$, then $\|v\|_{\mathrm{H}}=0$.

Proof. Consider a path $\left\{\eta_{t}\right\}_{(-1,1)} \subset \mathcal{H}_{d}(S)$ so that $\hat{\sigma}\left(\eta_{t}\right)=\eta_{-t}$ for all $t \in(-1,1)$. Then, $\ell_{\mathbf{H}}\left(\eta_{t}(\gamma)\right)=\ell_{\mathbf{H}}\left(\eta_{-t}(\gamma)\right)$ and $h\left(f_{\eta_{t}}^{\mathrm{H}}\right)=h\left(f_{\eta_{-t}}^{\mathrm{H}}\right)$ for all $t \in(-1,1)$ and $\gamma \in \pi_{1}(S)$. Therefore,

$$
\left.\frac{\partial}{\partial t}\right|_{t=0} h\left(f_{\eta_{t}}^{\mathrm{H}}\right) \ell_{f_{\eta_{t}}^{\mathrm{H}}}(\gamma)=0
$$

for all $\gamma \in \pi_{1}(S)$. Corollary 2.5 then implies that $\|v\|_{\mathrm{H}}=0$.

Remark: With a little more effort one may use the techniques of [17] to show that these are the only degenerate vectors for $\|\cdot\|_{H}$ and that $\|\cdot\|_{H}$ induces a path metric on the Hitchin component. 


\section{Generalizations and consequences}

In [17] we work in the more general setting of Anosov representations of word hyperbolic groups into semi-simple Lie groups. In this section, we will survey these more general results and discuss some of the additional difficulties which occur. The bulk of the work in [17] is done in the setting of projective Anosov representations into $\mathrm{SL}_{d}(\mathbb{R})$. We note that Hitchin representations are examples of projective Anosov representations as are Benoist representations, i.e. holonomy representations of closed strictly convex (real) projective manifolds (see Guichard-Wienhard [40, Prop. 6.1]).

6.1. Projective Anosov representations. We first show that the pressure form gives an analytic Riemannian metric on the space of (conjugacy classes of) projective Anosov, generic, regular $^{1}$, irreducible representations. In order to define projective Anosov representations, we begin by recalling basic facts about the geodesic flow of a word hyperbolic group.

Gromov [36] first established that a word hyperbolic group $\Gamma$ has an associated geodesic flow $U_{\Gamma}$. Roughly, one considers the obvious flow on the space of all geodesics in the Cayley graph of $\Gamma$, collapses all geodesics joining two points in the Gromov boundary to a single geodesic, and considers the quotient by the action of $\Gamma$. We make use of the version due to Mineyev [63] (see also Champetier [24]). Mineyev defines a proper cocompact action of $\Gamma$ on $\widetilde{U_{\Gamma}}=\partial_{\infty} \Gamma^{(2)} \times \mathbb{R}$ and a metric on $\widetilde{U_{\Gamma}}$, well-defined only up to Hölder equivalence, so that $\Gamma$ acts by isometries, every orbit of $\mathbb{R}$ is quasiisometrically embedded, and the $\mathbb{R}$-action is by Lipschitz homeomorphisms. Moreover, the $\mathbb{R}$-action descends to a flow on $U_{\Gamma}=\widetilde{U_{\Gamma}} / \Gamma$. In the case that $\Gamma$ is the fundamental group of a negatively curved manifold $M$, one may take $U_{\Gamma}$ to be the geodesic flow on $T^{1} M$.

A representation $\rho: \Gamma \rightarrow \mathrm{SL}_{d}(\mathbb{R})$ has transverse projective limit maps if there exist continuous, $\rho$-equivariant limit maps

$$
\xi_{\rho}: \partial_{\infty} \Gamma \rightarrow \mathbb{P}\left(\mathbb{R}^{d}\right)
$$

and

$$
\theta_{\rho}: \partial_{\infty} \Gamma \rightarrow G r_{d-1}\left(\mathbb{R}^{d}\right)=\mathbb{P}\left(\left(\mathbb{R}^{d}\right)^{*}\right)
$$

so that if $x$ and $y$ are distinct points in $\partial_{\infty} \Gamma$, then

$$
\xi_{\rho}(x) \oplus \theta_{\rho}(y)=\mathbb{R}^{d} .
$$

A representation $\rho$ with transverse projective limit maps determines a splitting $\Xi \oplus \Theta$ of the flat bundle $E_{\rho}$ over $U_{\Gamma}$. Concretely, if $\tilde{E}_{\rho}$ is the lifted bundle over $\widetilde{U_{\Gamma}}$, then the lift $\tilde{\Xi}$ of $\Xi$ has fiber $\xi_{\rho}(x)$ and the lift $\tilde{\Theta}$ of $\Theta$ has fiber $\theta_{\rho}(y)$ over the point $(x, y, t)$. The geodesic flow on $U_{\Gamma}$ lifts to a flow on $\widetilde{U_{\Gamma}}$ which extends, trivially in the bundle factor, to a flow on $\tilde{E}_{\rho}$

\footnotetext{
${ }^{1} \mathrm{~A}$ representation $\rho: \Gamma \rightarrow \mathrm{SL}_{d}(\mathbb{R})$ is regular if it is a smooth point of the algebraic variety $\operatorname{Hom}\left(\Gamma, \mathrm{SL}_{d}(\mathbb{R})\right)$.
} 
which descends to a flow on $E_{\rho}$. One says that $\rho$ is projective Anosov if the resulting flow on the associated bundle $\operatorname{Hom}(\Theta, \Xi)=\Xi \otimes \Theta^{*}$ is contracting.

Projective Anosov representations are discrete, well-displacing, quasi-isometric embeddings with finite kernel such that the image of each infinite order element is bi-proximal, i.e. its eigenvalues of maximal and minimal modulus have multiplicity one (see Labourie [50, 51] and Guichard-Wienhard [40, Thm. 5.3,5.9]). However, projective Anosov representations need not be irreducible and the images of elements need not be diagonalizable over $\mathbb{R}$. On the other hand, Guichard and Wienhard [40, Prop. 4.10] showed that any irreducible representation with transverse projective limits maps is projective Anosov.

6.2. Deformation spaces. The space of all projective Anosov representations of a fixed word hyperbolic group $\Gamma$ into $\mathrm{SL}_{d}(\mathbb{R})$ is an open subset of $\operatorname{Hom}\left(\Gamma, \mathrm{SL}_{d}(\mathbb{R})\right) / \mathrm{SL}_{d}(\mathbb{R})$ (see Labourie [50, Prop. 2.1] and GuichardWienhard [40, Thm. 5.13]). However, a projective Anosov representation need not be a smooth point of $\operatorname{Hom}\left(\Gamma, \mathrm{SL}_{d}(\mathbb{R})\right) / \mathrm{SL}_{d}(\mathbb{R})$ (see Johnson-Millson [46]). Moreover, the set of projective Anosov representations need not be an entire component of $\operatorname{Hom}\left(\Gamma, \mathrm{SL}_{d}(\mathbb{R})\right) / \mathrm{SL}_{d}(\mathbb{R})$.

In order to have the structure of a real analytic manifold, we consider the space $\widetilde{\mathcal{C}}(\Gamma, d)$ of regular, projective Anosov, irreducible representations $\rho: \Gamma \rightarrow \mathrm{SL}_{d}(\mathbb{R})$ and let

$$
\mathcal{C}(\Gamma, d)=\widetilde{\mathcal{C}}(\Gamma, d) / \mathrm{SL}_{d}(\mathbb{R}) .
$$

If $\mathrm{G}$ is a reductive subgroup of $S \mathrm{~L}_{d}(\mathbb{R})$, we can restrict the whole discussion to representations with image in $G$, i.e. let $\widetilde{\mathcal{C}}(\Gamma, G)$ be the space of regular, projective Anosov, irreducible representations $\rho: \Gamma \rightarrow \mathrm{G}$ and let

$$
\mathcal{C}(\Gamma, G)=\widetilde{\mathcal{C}}(\Gamma, G) / G
$$

We will later want to restrict to the space $\mathcal{C}_{g}(\Gamma, \mathrm{G})$ of $\mathrm{G}$-generic representation in $\mathcal{C}(\Gamma, G)$, i.e. representations such that the centralizer of some element in the image is a maximal torus in $G$. In particular, in the case that $\mathrm{G}=\mathrm{SL}_{d}(\mathbb{R})$, a representation is $\mathrm{G}$-generic if some element in the image is diagonalizable over $\mathbb{C}$ with distinct eigenvalues. The resulting spaces are real analytic manifolds.

Proposition 6.1. ([17, Prop. 7.1]) If $\Gamma$ is a word hyperbolic group and $\mathrm{G}$ is a reductive subgroup of $\mathrm{SL}_{d}(\mathbb{R})$, then $\mathcal{C}(\Gamma, d), \mathcal{C}(\Gamma, \mathrm{G}), \mathcal{C}_{g}(\Gamma, \mathrm{G})$ and $\mathcal{C}_{g}(\Gamma, d)=\mathcal{C}_{g}\left(\Gamma, \mathrm{SL}_{d}(\mathbb{R})\right)$ are all real analytic manifolds.

6.3. The geodesic flow, entropy and intersection number. One new difficulty which arises, is that it is not known in general whether or not the geodesic flow of a word hyperbolic group is metric Anosov, i.e. a Smale flow in the sense of Pollicott [68]. Notice that our construction in Section 5.2 immediately generalizes to give, for any projective Anosov representation $\rho$, a geodesic flow $U_{\rho}$ which is Hölder orbit equivalent to $U_{\Gamma}$ and whose periods are exactly spectral radii of infinite order elements of $\Gamma$. In general, we must 
further show [17, Prop. 5.1] that $U_{\rho}$ is a topologically transitive metric Anosov flow.

Proposition 6.2. ([17, Prop. 4.1, 5.1]) If $\rho: \Gamma \rightarrow \mathrm{SL}_{d}(\mathbb{R})$ is projective Anosov, then there exists a topologically transitive, metric Anosov flow $U_{\rho}$ which is Hölder orbit equivalent to $U_{\Gamma}$ such that the orbit associated to $\gamma \in \Gamma$ has period $\Lambda(\rho(\gamma))$.

Lemma 2.2 provides a Hölder function $f_{\rho}: \mathrm{U}_{\Gamma} \rightarrow(0, \infty)$, well-defined up to Livšic cohomology, such that $U_{\rho}$ is Hölder conjugate to the reparametrization of $U_{\Gamma}$ by $f_{\rho}$. One may then use the Thermodynamic Formalism to define the entropy of a projective Anosov representation and the intersection number and renormalized intersection number of two projective Anosov representations. If $\rho$ is projective Anosov, we define

$$
R_{T}(\rho)=\left\{[\gamma] \in\left[\pi_{1}(S)\right] \mid \log \left(\Lambda_{\gamma}(\rho)\right) \leq T\right\}
$$

and the entropy of $\rho$ is given by

$$
h(\rho)=h\left(f_{\rho}\right)=\lim _{T \rightarrow \infty} \frac{\log \# R_{T}(\rho)}{T} .
$$

The intersection number of two projective Anosov representations $\rho$ and $\eta$ is given by

$$
\mathbf{I}(\rho, \eta)=\mathbf{I}\left(f_{\rho}, f_{\eta}\right)=\lim _{T \rightarrow \infty} \frac{1}{\# R_{T}(\rho)} \sum_{[\gamma] \in R_{\rho}(T)} \frac{\log \left(\Lambda_{\gamma}(\eta)\right)}{\log \left(\Lambda_{\gamma}(\rho)\right)}
$$

and their renormalized intersection number is

$$
\mathbf{J}(\rho, \eta)=\frac{h(\eta)}{h(\rho)} \mathbf{I}(\rho, \eta) .
$$

One may use the technique of proof of Proposition 5.9 to show that all these quantities vary analytically.

Theorem 6.3. ([17, Thm. 1.3]) If $\Gamma$ is a word hyperbolic group and $\mathrm{G}$ is a reductive subgroup of $\mathrm{PSL}_{\mathrm{d}}(\mathbb{R})$, then entropy varies analytically over $\mathcal{C}(\Gamma, G)$ and intersection number and renormalized intersection number vary analytically over $\mathcal{C}(\Gamma, G) \times \mathcal{C}(\Gamma, G)$.

6.4. The pressure metric for projective Anosov representation spaces. If $G$ is a reductive subgroup of $P S L_{d}(\mathbb{R})$, we define a thermodynamic mapping

$$
\Phi: \mathcal{C}(\Gamma, \mathrm{G}) \rightarrow \mathcal{H}\left(\mathrm{U}_{\Gamma}\right)
$$

by $\rho \mapsto\left[-h\left(f_{\rho}\right) f_{\rho}\right]$. We can again show that $\Phi$ has locally analytic lifts, so we can pull back the pressure norm on $\mathcal{P}\left(U_{\Gamma}\right)$ to obtain a pressure seminorm $\|\cdot\|_{\mathbf{P}}$ on $\mathcal{C}(\Gamma, G)$. The resulting pressure semi-norm gives an analytic Riemannian metric on $\mathcal{C}_{g}(\Gamma, \mathrm{G})$.

Theorem 6.4. ([17, Thm. 1.4]) If $\Gamma$ is a word hyperbolic group and $\mathrm{G}$ is a reductive subgroup of $\mathrm{SL}_{d}(\mathbb{R})$, then the pressure form is an analytic $\operatorname{Out}(\Gamma)$ invariant Riemannian metric on $\mathcal{C}_{g}(\Gamma, \mathrm{G})$. In particular, the pressure form is an analytic $\operatorname{Out}(\Gamma)$-invariant Riemannian metric on $\mathcal{C}_{g}(\Gamma, d)$. 
It only remains to prove that the pressure semi-norm is non-degenerate. We follow the same outline as in the Hitchin setting, but encounter significant new technical difficulties. As before, we may use Corollary 2.5 to obtain restrictions on the derivatives of spectral length of group elements.

Lemma 6.5. ([17, Lem. 9.3]) If $\mathrm{G}$ is a reductive subgroup of $\mathrm{PSL}_{\mathrm{d}}(\mathbb{R})$, $v \in \mathrm{T}_{\rho} \mathcal{C}(\Gamma, \mathrm{G})$ and $\|v\|_{\mathbf{P}}=0$, then

$$
D_{\rho} \log \Lambda_{\gamma}(v)=-\frac{D_{\rho} h(v)}{h(\rho)} \log \Lambda_{\gamma}(\rho)
$$

for all $\gamma \in \Gamma$.

We use this to establish the following analogue of Proposition 5.13 from the Hitchin setting. In order to do so, we must work in the setting of G-generic representations and we can only conclude that the derivative of spectral length, rather than trace, is trivial.

Proposition 6.6. ([17, Prop 9.1]) If $\mathrm{G}$ is a reductive subgroup of $\mathrm{PSL}_{\mathrm{d}}(\mathbb{R})$, $v \in \mathrm{T}_{\rho} \mathcal{C}_{g}(\Gamma, \mathrm{G})$ and there exists $K$ such that

$$
D_{\rho} \log \Lambda_{\gamma}(v)=K \log \Lambda_{\gamma}(\rho)
$$

for all $\gamma \in \Gamma$, then $K=0$. In particular, $D_{\rho} \log \Lambda_{\gamma}(v)=0$ for all $\gamma \in \Gamma$.

One completes the proof by showing that the derivatives of the spectral radii functions generate the cotangent space.

Proposition 6.7. ([17, Prop. 10.3]) If $\mathrm{G}$ is a reductive subgroup of $\mathrm{PSL}_{\mathrm{d}}(\mathbb{R})$ and $\rho \in \mathcal{C}_{g}(\Gamma, \mathrm{G})$, then the set $\left\{D_{\rho} \Lambda_{\gamma} \mid \gamma \in \Gamma\right\}$ spans $\mathrm{T}_{\rho}^{*} \mathcal{C}_{g}(\Gamma, \mathrm{G})$.

6.5. Anosov representations. We now discuss the generalizations of our work to spaces of more general Anosov representations. If $G$ is any semisimple Lie group with finite center and $\mathrm{P}^{ \pm}$is a pair of opposite parabolic subgroups, then one may consider $\left(G, P^{ \pm}\right)$-Anosov representations of a word hyperbolic group $\Gamma$ into $\mathrm{G}$. A $\left(\mathrm{G}, \mathrm{P}^{ \pm}\right)$-Anosov representation $\rho: \Gamma \rightarrow \mathrm{G}$ has limit maps

$$
\xi_{\rho}^{ \pm}: \partial_{\infty} \Gamma \rightarrow \mathrm{G} / \mathrm{P}^{ \pm}
$$

(which are transverse in an appropriate sense and give rise to associated flows with contracting/dilating properties). In fact, Zariski dense representations with transverse limit maps are always $\left(G, P^{ \pm}\right)$-Anosov ([40, Thm 4.11]).

Projective Anosov representations are $\left(\mathrm{G}, \mathrm{P}^{ \pm}\right)$-Anosov where $\mathrm{G}=\mathrm{SL}_{d}(\mathbb{R})$, $\mathrm{P}^{+}$is the stabilizer of a line and $\mathrm{P}^{-}$is the stabilizer of a complementary hyperplane ([17, Prop. 2.11]). Hitchin representations are $\left(\mathrm{G}, \mathrm{P}^{ \pm}\right)$-Anosov where $G=S L_{d}(\mathbb{R}), \mathrm{P}^{+}$is the group of upper triangular matrices (i.e. the stabilizer of the standard flag) and $\mathrm{P}^{-}$is the group of lower triangular matrices (Labourie [50]).

We may think of Anosov representations as natural generalizations of Fuchsian representations, since they are discrete, faithful, quasi-isometric embeddings with finite kernel so that the image of every infinite order element is $\mathrm{P}^{+}$-proximal $([50,51]$ and $[40$, Thm. 5.3,5.9]). More generally, 
they may be thought of as generalizations of convex cocompact representations into rank one Lie groups. See Labourie [50] and Guichard-Wienhard [40] for definitions and more detailed discussions of Anosov representations. Gueritaud-Guichard-Kassel-Wienhard [37] and Kapovich-Leeb-Porti [47] have developed intriguing new viewpoints on Anosov representations and their definition.

Guichard and Wienhard [40, Prop. 4.2, Remark 4.12] (see also [17, Thm 2.12]) showed that there exists an irreducible representation $\sigma: \mathrm{G} \rightarrow \mathrm{SL}(V)$ (called the Plücker representation) such that $\rho: \Gamma \rightarrow \mathrm{G}$ is $\left(\mathrm{G}, \mathrm{P}^{ \pm}\right)$-Anosov if and only if $\sigma \circ \rho$ is projective Anosov. Thus, one can often reduce the study of $\left(\mathrm{G}, \mathrm{P}^{ \pm}\right)$-Anosov representations to the study of projective Anosov representations.

Let $\mathcal{Z}\left(\Gamma, \mathrm{G}, \mathrm{P}^{ \pm}\right)$be the space of (conjugacy classes of) regular, virtually Zariski dense $\left(\mathrm{G}, \mathrm{P}^{ \pm}\right)$-Anosov representations. The space $\mathcal{Z}\left(\Gamma, \mathrm{G}, \mathrm{P}^{ \pm}\right)$is an analytic orbifold, which is a manifold if $\mathrm{G}$ is connected (see [17, Prop. 7.3]). The Plücker representation $\sigma: \mathrm{G} \rightarrow \mathrm{SL}_{d}(\mathbb{R})$ allows one to view $\mathcal{Z}\left(\Gamma, \mathrm{G}, \mathrm{P}^{ \pm}\right)$ as an analytically varying family of $\sigma(\mathrm{G})$-generic projective Anosov representations. One may pull back the pressure form and adapt the techiques from the projective Anosov setting to prove:

Theorem 6.8. ([17, Cor. 1.9]) If $\mathrm{G}$ is semi-simple Lie group with finite center and $\Gamma$ is word hyperbolic, then the pressure form is an Out $(\Gamma)$-invariant analytic Riemannian metric on $\mathcal{Z}\left(\Gamma, \mathrm{G}, \mathrm{P}^{ \pm}\right)$.

6.6. Examples. There are two other important classes of higher Teichmüller spaces which are (quotients of) entire components of representation varieties.

Burger, Iozzi and Wienhard [21] have studied representations of $\pi_{1}(S)$ into a Hermitian Lie group $G$ of tube type with maximal Toledo invariant, i.e. maximal representations. Each maximal representation is Anosov, with respect to stabilizers of points in the Shilov boundary of the associated symmetric space $([20,22])$, and the space of all maximal representations is a collection of components of $\operatorname{Hom}\left(\pi_{1}(S), \mathrm{G}\right)([21])$. One particularly nice case arises when $G=\operatorname{Sp}(4, \mathbb{R})$, in which case there are $2 g-3$ components which are non-simply connected manifolds consisting entirely of Zariski dense representations (see Bradlow-Garcia-Prada-Gothen [13]). Hence, the quotients by $\mathrm{G}$ of all such components admit pressure metrics.

Benoist $[5,6]$ studied holonomies of strictly convex projective structures on a closed manifold $M$ and showed that these consist of entire components of $\operatorname{Hom}\left(\pi_{1}(M), \mathrm{PSL}_{\mathrm{d}}(\mathbb{R})\right)$. One may use his work to show that these representations, which we call Benoist representations, are projective Anosov (see Guichard-Wienhard [40, Prop. 6.1]). Johnson-Millson [46] gave examples of holonomy maps $\rho: \pi_{1}(M) \rightarrow \mathrm{SO}(d-1,1)$ of closed hyperbolic $d$-1-manifold, where $d \geq 5$, such that $\rho$ is a singular point of $\operatorname{Hom}\left(\pi_{1}(M), \operatorname{PSL}_{d}(\mathbb{R})\right)$.

6.7. Rank one Lie groups. Let $\Gamma$ be a word hyperbolic group and let $\mathrm{G}$ be a rank 1 semi-simple Lie group, e.g. $\operatorname{PSL}_{2}(\mathbb{C})$. A representation $\rho: \Gamma \rightarrow G$ 
is convex cocompact if and only if whenever one chooses a basepoint $x_{0}$ for the symmetric space $X=\mathrm{K} \backslash \mathrm{G}$ then the orbit map $\tau: \Gamma \rightarrow X$ given by $\gamma \rightarrow \gamma\left(x_{0}\right)$ is a quasi-isometric embedding. The limit set of $\rho(\Gamma)$ is then the set of accumulation points in $\partial_{\infty} X$ of the image of the orbit map and one can define the Hausdorff dimension of this set. Patterson [67], Sullivan [81], Corlette-Iozzi [26], and Yue [93] showed that the topological entropy of a convex cocompact representation agrees with the Hausdorff dimension of the limit set of its image.

A representation $\rho: \Gamma \rightarrow \mathrm{G}$ is convex cocompact if and only if it is Anosov (see Guichard-Wienhard [40, Thm. 5.15]). Since the Plücker embedding multiplies entropy by a constant depending only on G (see [17, Cor. 2.14]), the analyticity of the Hausdorff dimension of the limit set follows from the analyticity of entropy for projective Anosov representations.

Theorem 6.9. ([17, Cor. 1.8]) If $\Gamma$ is a word hyperbolic group and $\mathrm{G}$ is a rank 1 semi-simple Lie group, then the Hausdorff dimension of the limit set varies analytically over analytic families of convex cocompact representations of $\Gamma$ into $\mathrm{G}$.

Remark: When $\mathrm{G}=\mathrm{PSL}_{2}(\mathbb{C})$, Ruelle [72] proved this for surface groups and Anderson-Rocha [2] proved it for free products of surface groups and free groups. Tapie [82] used work of Katok-Knieper-Pollicott-Weiss [49] to show that the Hausdorff dimension is $C^{1}$ on smooth families of convex cocompact representations.

Let $C C\left(\Gamma, \mathrm{PSL}_{2}(\mathbb{C})\right)$ be the space of (conjugacy classes of ) convex cocompact representation of $\Gamma$ into $\mathrm{PSL}_{2}(\mathbb{C})$. Bers $[7]$ showed that $C C\left(\Gamma, \mathrm{PSL}_{2}(\mathbb{C})\right)$ is an analytic manifold. Recall that a convex cocompact representation is not Zariski dense if and only if it is virtually Fuchsian, i.e. contain a finite index subgroup conjugate into $\mathrm{PSL}_{2}(\mathbb{R})$. We may again use the Plücker representation to prove:

Theorem 6.10. ([17, Cor. 1.7]) If $\Gamma$ is word hyperbolic, then the pressure form is $\operatorname{Out}(\Gamma)$-invariant and analytic on $C C\left(\Gamma, \mathrm{PSL}_{2}(\mathbb{C})\right)$ and is nondegenerate at any representation which is not virtually Fuchsian. In particular, if $\Gamma$ is not either virtually free or virtually a surface group, then the pressure form is an analytic Riemannian metric on $C C\left(\Gamma, \mathrm{PSL}_{2}(\mathbb{C})\right)$. Moreover, the pressure form always induces a path metric on $C C\left(\Gamma, \mathrm{PSL}_{2}(\mathbb{C})\right)$.

Bridgeman [14] had previously defined and studied the pressure metric on quasifuchsian space $Q F(S)=C C\left(\pi_{1}(S), \mathrm{PSL}_{2}(\mathbb{C})\right)$. He showed that the degenerate vectors in this case correspond exactly to pure bending vectors on the Fuchsian locus.

6.8. Margulis space times. A Margulis space time is a quotient of $\mathbb{R}^{3}$ by a free, non-abelian group of affine transformations which acts properly discontinuously on $\mathbb{R}^{3}$. They were originally discovered by Margulis [59] as counterexamples to a question of Milnor. Ghosh [32] used work of Goldman, 
Labourie and Margulis [34, 35] to interpret holonomy maps of Margulis space times (without cusps) as "Anosov representations" into the (non-semisimple) Lie group $\operatorname{Aff}\left(\mathbb{R}^{3}\right)$ of affine automorphisms of $\mathbb{R}^{3}$. Ghosh [33] was then able to adapt the techniques of [17] to produce a pressure form on the analytic manifold $\mathcal{M}$ of (conjugacy classes of) holonomy maps of Margulis space times of fixed rank (with no cusps). This pressure form is an analytic Riemannian metric on the slice $\mathcal{M}_{k}$ of $\mathcal{M}$ consisting of holonomy maps with entropy $k$ (see Ghosh [33, Thm. 1.0.1]), but has a degenerate direction on $\mathcal{M}$, so the pressure form has signature $(\operatorname{dim} \mathcal{M}-1,0)$ on $\mathcal{M}$.

\section{Open PROBLEMS}

The geometry of the pressure metric is still rather mysterious and much remains to be explored. The hope is that the geometry of the pressure metric will yield insights into the nature of the Hitchin component and other higher Teichmüller spaces, in much the way that the study of the Teichmüller and Weil-Petersson metrics have been an important tool in our understanding of Teichmüller space and the mapping class group. It is natural to begin by exploring analogies with the Weil-Petersson metric on Teichmüller space. We begin the discussion by recalling some basic properties of the Weil-Petersson metric.

\section{Properties of the Weil-Petersson metric:}

(1) The extended mapping class group is the isometry group of $\mathcal{T}(S)$ in the Weil-Petersson metric (Masur-Wolf [61]).

(2) The Weil-Petersson metric is negatively curved, but the sectional curvature is not bounded away from either 0 or $-\infty$ (Wolpert [89], Tromba [85], Huang [43]).

(3) If $\phi$ is a pseudo-Anosov mapping class, then there is a lower bound for its translation distance on Teichmüller space and there is a unique invariant geodesic axis for $\phi$ (Daskalopoulos-Wentworth [30]).

(4) The Weil-Petersson metric is incomplete (Wolpert [88], Chu [25]). However, it admits a metric completion which is $C A T(0)$ and homeomorphic to the augmented Teichmüller space (see Masur [60] and Wolpert [91]).

Masur and Wolf's result [61] on the isometry group of $\mathcal{T}(S)$ suggests the following problem.

Problem 1: Is the isometry group of a Hitchin component generated by the (extended) mapping class group and the contragredient involution? More generally, explore whether the relevant outer automorphism group is a finite index subgroup of the isometry group of a higher Teichmüller space with the pressure metric.

Bridgeman, Canary, and Labourie [16] have shown that any diffeomorphism of $\mathcal{H}_{3}(S)$ which preserves the intersection number is an element of the extended mapping class group or the composition of an element in the 
extended mapping class group with the contragredient involution. Along the way, they show that any diffeomorphism which preserves the intersection number also preserves the entropy and hence preserves the renormalized intersection number, the pressure metric, and, by work of Potrie and Sambarino [71], the Fuchsian locus. This suggests the following problem:

Problem 2: Prove that if $g: \mathcal{H}_{d}(S) \rightarrow \mathcal{H}_{d}(S)$ is an isometry with respect to the pressure metric then $\mathbf{I}(g(\rho), g(\sigma))=\mathbf{I}(\rho, \sigma)$ for all $\rho, \sigma \in \mathcal{H}_{d}(S)$. It would follow that the isometry of the group of the Hitchin component $\mathcal{H}_{3}(S)$ is generated by the extended mapping class group of $S$ and the contragredient involution.

Bridgeman and Canary [15] have shown that the group of diffeomorphisms of quasifuchsian space $Q F(S)$ which preserve the renormalized intersection number is generated by the extended mapping class group and complex conjugation. So one may also consider the corresponding analogue of Problem 2 in quasifuchsian space.

It would be be useful to study the curvature of the pressure metric, guided by the results of Wolpert [89], Tromba [85], and Huang [43]. Wolf's work [87] (see also [86]) on the Hessians of length functions on Teichmüller space may offer a plan of attack here.

Problem 3: Investigate the curvature of the Hitchin component in the pressure metric.

Pollicott and Sharp [70] have investigated the curvature of the pressure metric on deformation spaces of marked metric graphs with entropy 1 . In this setting, the curvature can be both positive and negative.

Labourie and Wentworth [52] have derived a formula for the pressure metric at points in the Fuchsian locus of a Hitchin component in terms of Hitchin's parameterization of the Hitchin component by holomorphic differentials. They also obtain variational formulas which are analogues of classical results in the Teichmüller setting.

Since Labourie [51] proved that the mapping class group acts properly discontinuously on a Hitchin component, it is natural to study the geometry of this action. One specific question to start with would be:

Problem 4: Is there a lower bound for the translation distance for the action of a pseudo-Anosov mapping class on the Hitchin component?

Since the restriction of the pressure metric to the Fuchsian locus is a multiple of the Weil-Petersson metric, the Hitchin component is incomplete and the metric completion contains augmented Teichmüller space. However, very little is known about the completeness of the pressure metric in "other directions." The work of Zhang [94, 95] and Loftin [56] (when $d=3$ ) should be relevant here. It may also be interesting to study the relationship between the metric completion and Parreau's compactification [65] of the Hitchin component. 
Problem 5: Investigate the metric completion of the Hitchin component or other higher Teichmüller spaces.

$\mathrm{Xu}$ [92] studied the pressure metric on the Teichmuller space $\mathcal{T}(S)$ where $S$ is a surface with non-empty geodesic boundary. He shows that the pressure metric in this case is not equal to the classical Weil-Petersson metric on $\mathcal{T}(S)$. He further shows that it is not complete and that the space of marked metric graphs on a fixed graph with its pressure metric arises naturally in the completion.

The following problem indicates how little is known about the coarse geometry of the pressure metric. We recall that a subset $A$ of a metric space $X$ is said to be coarsely dense if there exists $D>0$ such that every point in $X$ lies within $D$ of a point in $A$.

Problem 6: (a) Is the Fuchsian locus coarsely dense in a Hitchin component?

(b) Is the Fuchsian locus coarsely dense in quasifuchsian space?

(c) If $M$ is an acylindrical 3-manifold with no toroidal boundary components and $\Gamma=\pi_{1}(M)$, does $C C\left(\Gamma, \mathrm{PSL}_{2}(\mathbb{C})\right)$ have finite diameter?

Zhang [94, 95] and Nie [64] (when $d=3$ ) produce sequences in Hitchin components where entropy converges to 0 . These sequences are candidates to produce points arbitrarily far from the Fuchsian locus.

In case (c), Out $(\Gamma)$ is finite (see Johannson [45]) and $C C\left(\Gamma, \mathrm{PSL}_{2}(\mathbb{C})\right.$ ) has compact closure in the $\mathrm{PSL}_{2}(\mathbb{C})$-character variety (see Thurston [84]).

One may phrase all the above questions as being about the quotient of a higher Teichmüller space by its natural automorphism group. Similarly, one might ask whether the quotient of the Hitchin component by the mapping class group has finite volume.

Problem 7: Does the quotient of the Hitchin component by the action of the mapping class group have finite volume in the quotient pressure metric?

Potrie-Sambarino [71] showed that the entropy function is maximal uniquely on the Fuchsian locus of a Hitchin component, so it is natural to investigate more subtle behavior of the entropy function.

Problem 8: Investigate the critical points on the entropy function.

Bowen [10] showed that the entropy function is uniquely minimal on the Fuchsian locus in quasifuchsian space $Q F(S)$. Bridgeman [14] showed that the entropy function on $Q F(S)$ has no local maxima and moreover the Hessian of the entropy function is positive-definite on at least a half-dimensional subspace at any critical point.

If $M$ is an acylindrical 3-manifold with no toroidal boundary components and $\Gamma=\pi_{1}(M)$, then there is a unique representation in $C C\left(\Gamma, \mathrm{PSL}_{2}(\mathbb{C})\right)$ where the boundary of the limit set of the image consists of round circles (see Thurston [83]). It is conjectured that the entropy has a unique minimum at this representation (see Canary-Minsky-Taylor [23]). Storm [80] proved 
that this is the unique representation where the volume of the convex core achieves its minimum.

In the case of $C C\left(\Gamma, \mathrm{PSL}_{2}(\mathbb{C})\right)$ we were able to obtain a path metric, even when the pressure form is degenerate on a submanifold. One might hope to be able to do so in more general settings.

Problem 9: If $\Gamma$ is a word hyperbolic group, $\mathrm{G}$ is a semisimple Lie group and $\mathrm{P}^{ \pm}$is a pair of opposite parabolic subgroups, can one extend the pressure metric on $\mathcal{Z}\left(\Gamma, \mathrm{G}, \mathrm{P}^{ \pm}\right.$) to a path metric on the space of all (conjugacy classes of ) $\left(\mathrm{G}, \mathrm{P}^{ \pm}\right)$-Anosov representations of $\Gamma$ into $\mathrm{G}$ ?

\section{REFERENCES}

[1] L. Ahlfors and A. Beurling, "The boundary correspondence under quasiconformal mappings," Acta Math. 96(1956), 125-142.

[2] J.W. Anderson and A. Rocha, "Analyticity of Hausdorff dimension of limit sets of Kleinian groups," Ann. Acad. Sci. Fenn. 22(1997), 349-364.

[3] Y. Benoist, "Propriétés asymptotiques des groupes linéaires," G.A.F.A. 7(1997), 1-47.

[4] Y. Benoist, "Propriétés asymptotiques des groupes linéaires II," Adv. Stud. Pure Math. 26(2000), 33-48.

[5] Y. Benoist, "Convexes divisibles I," in Algebraic groups and arithmetic, Tata Inst. Fund. Res. Stud. Math. 17(2004), 339-374.

[6] Y. Benoist, "Convexes divisibles III," Ann. Sci. de l'E.N.S. 38(2005), 793-832.

[7] L. Bers, "Spaces of Kleinian groups," in Maryland conference in Several Complex Variables I, Springer-Verlag Lecture Notes in Math, No. 155(1970), 9-34.

[8] F. Bonahon, "The geometry of Teichmüller space via geodesic currents," Invent. Math. 92(1988), 139-162.

[9] R. Bowen, "Periodic orbits of hyperbolic flows," Amer. J. Math. 94(1972), 1-30.

[10] R. Bowen, "Hausdorff dimension of quasi-circles," Publ. Math. de l'I.H.E.S. 50(1979), 1125.

[11] R. Bowen, Equilibrium States and the Ergodic Theory of Anosov Diffeomorphisms, Springer-Verlag Lecture Notes in Mathematics, vol. 470, 1975.

[12] R. Bowen and D. Ruelle, "The ergodic theory of axiom A flows," Invent. Math. 29(1975), 181-202.

[13] S.Bradlow, O. Garcia-Prada and P. Gothen, "Deformations of maximal representations in $\mathrm{Sp}(4, \mathbb{R}), "$ Quart. J. Math. 63(2012), 795-843.

[14] M. Bridgeman, "Hausdorff dimension and the Weil-Petersson extension to quasifuchsian space," Geom. and Top. 14(2010), 799-831.

[15] M. Bridgeman and R. Canary, "Simple length rigidity for Kleinian surface groups and applications," preprint, available at: https://arxiv.org/abs/1509.02510

[16] M. Bridgeman, R. Canary and F. Labourie, "Simple length rigidity for Hitchin representations," preprint available at: http://front.math.ucdavis.edu/1703.07336.

[17] M. Bridgeman, R. Canary, F. Labourie and A. Sambarino, "The pressure metric for Anosov representations," G.A.F.A. 25(2015), 1089-1179.

[18] M. Bridgeman and E. Taylor, "An extension of the Weil-Petersson metric to quasi-fuchsian space," Mathematische Annalen, 341(2008), 927-943.

[19] M. Burger, "Intersection, the Manhattan curve and Patterson-Sullivan theory in rank 2," Internat. Math. Res. Notices 7(1993), 217-225.

[20] M. Burger, A. Iozzi, F. Labourie and A. Wienhard, "Maximal representations of surface groups: symplectic Anosov structures," P.A.M.Q. 1(2005), 555-601.

[21] M. Burger, A. Iozzi, and A. Wienhard, "Surface group representations with maximal Toledo invariant," Annals of Math. 172(2010), 517-566.

[22] M. Burger, A. Iozzi and A. Wienhard, "Maximal representations and Anosov structures," in preparation.

[23] R.D. Canary, Y.N. Minsky and E.C. Taylor, "Spectral theory, Hausdorff dimension and the topology of hyperbolic 3-manifolds," J. Geom. Anal. 9(1999), 17-40. 
[24] C. Champetier, "Petite simplification dans les groupes hyperboliques," Ann. Fac. Sci. Toulouse Math. 3(1994), 161-221.

[25] T. Chu, "The Weil-Petersson metric in moduli space," Chinese J. Math. 4(1976), 29-51.

[26] K.Corlette and A. Iozzi, "Limit sets of discrete groups of isometries of exotic hyperbolic spaces," Trans. A.M.S. 351(1999), 1507-1530.

[27] M. Crampon, "Entropies of compact strictly convex projective manifolds," J. Mod. Dyn. 3(2009), 511-547.

[28] F. Dal'Bo and I. Kim, "A criterion of conjugacy for Zariski dense subgroups," Comptes Rendus Math. 330 (2000), 647-650.

[29] M. Darvishzadeh and W. Goldman, "Deformation spaces of convex real projective structures and hyperbolic structures," J. Kor. Math. Soc. 33(1996), 625-639.

[30] G. Daskalopoulos and R. Wentworth, "Classification of Weil-Petersson isometries," Amer. J. Math. 125(2003), 941-975.

[31] V. Fock and A. Goncharov, "Moduli spaces of local systems and higher Teichmüller theory," Publ. Math. I.H.E.S. 103(2006), 1-211.

[32] S. Ghosh, "Anosov structure on Margulis space time," Groups Geom. Dyn., to appear, preprint available at: http://front.math.ucdavis.edu/1412.8211

[33] S. Ghosh, "The pressure metric on the Margulis multiverse," Geom. Ded., to appear, preprint available at: http://front.math.ucdavis.edu/1505.00534

[34] W. Goldman and F. Labourie, "Geodesics in Margulis spacetimes" Erg. Thy. Dyn. Sys. 32(2012), 643-651.

[35] W. Goldman, F. Labourie and G. Margulis, "Proper affine actions and geodesic flows of hyperbolic surfaces" Annals of Math. 170(2009), 1051-1083.

[36] M. Gromov, "Hyperbolic groups," in Essays in Group Theory, MSRI Publ. 8 (1987), 75-263.

[37] F.Gueritaud, O. Guichard, F. Kassel and A. Wienhard, "Anosov representations and proper actions," Geom. Top., to appear, preprint available at: http://front.math.ucdavis.edu/1502.03811

[38] O. Guichard, "Composantes de Hitchin et représentations hyperconvexes de groupes de surface," J. Diff. Geom. 80(2008), 39-431.

[39] O. Guichard, "Zariski closure of positive and maximal representation," in preparation.

[40] O. Guichard and A. Wienhard, "Anosov representations: Domains of discontinuity and applications," Invent. Math. 190(2012), 357-438.

[41] M. W. Hirsch, C. C. Pugh and M. Shub, Invariant manifolds Lecture Notes in Mathematics, Vol. 583, 1977

[42] N. Hitchin, "Lie groups and Teichmüller space," Topology 31(1992), 449-473.

[43] Z. Huang, "Asymptotic flatness of the Weil-Petersson metric on Teichmüller space," Geom. Ded. 110(2005), 81-102.

[44] J. Humphreys. Introduction to Lie algebras and representation theory, Springer-Verlag, 1972.

[45] K. Johannson, Homotopy Equivalences of 3-manifolds with Boundary, Lecture Notes in Mathematics, vol. 761, Springer-Verlag, 1979.

[46] D. Johnson and J. Millson, "Deformation spaces associated to compact hyperbolic manifolds," in Discrete Groups and Geometric Analysis, Progress in Math., vol. 67(1987), 48-106.

[47] M. Kapovich, B. Leeb and J. Porti, "Morse actions of discrete groups on symmetric spaces," http://front.math.ucdavis.edu/1403.7671

[48] A. Katok and B. Hasselblatt, Introduction to the modern theory of dynamical systems, Cambridge University Press, 1995.

[49] A. Katok, G. Knieper, M. Pollicott and H. Weiss, "Differentiability and analyticity of topological entropy for Anosov and geodesic flows," Invent. Math. 98(1989), 581-597.

[50] F. Labourie, "Anosov flows, surface groups and curves in projective space," Invent. Math. 165(2006), 51-114

[51] F. Labourie, "Cross ratios, Anosov representations and the energy functional on Teichmüller space," Ann. Sci. E.N.S. 41(2008), 437-469.

[52] F. Labourie and R. Wentworth "Variations along the fuchsian locus," Ann. Sci. E.N.S., to appear, preprint, available at: http://front.math.ucdavis.edu/1506.01686

[53] F. Ledrappier, "Structure au bord des variétés à courbure négative," Séminaire de théorie specrale et géométrie de Grenoble 13(1995), 97-122. 
[54] Q. Li, "Teichmüller space is totally geodesic in Goldman space," Asian J. Math., 20(2016), $21-46$.

[55] A.N. Livšic, "Cohomology of dynamical systems," Math. USSR Izvestija 6(1972), 1296-1320.

[56] J. Loftin, "The compactification of the moduli space of convex RP ${ }^{2}$ surfaces. I," J. Diff. Geom. 68(2004), 223-276.

[57] A. Lubotzky and A. Magid, Varieties of representations of finitely generated groups, Mem. Amer. Math. Soc. 58(1985), no. 336.

[58] A. Manning, "Topological entropy for geodesic flows," Annals of Math. 110(1979), 567-573.

[59] G. Margulis, "Free completely discontinuous groups of affine transformations," Soviet. Math. Dokl. 28(1983), 435-439.

[60] H. Masur, "Extension of the Weil-Petersson metric to the boundary of Teichmüller space," Duke Math. J. 43(1976), 623-635.

[61] H. Masur and M. Wolf, "The Weil-Petersson Isometry Group," Geom. Ded. 93(2002), 177190.

[62] C. McMullen, "Thermodynamics, dimension and the Weil-Petersson metric," Invent. Math. 173(2008), 365-425.

[63] I. Mineyev, "Flows and joins of metric spaces," Geom. Top. 9(2005), 403-482.

[64] X. Nie, "On the Hilbert geometry of simplicial Tits sets," Ann. Inst. Four. 65(2015), 10051030.

[65] A. Parreau, "Compactification d'espaces de représentations de groupes de type fini," Math. Z. 272(2012), 51-86.

[66] W. Parry and M. Pollicott, Zeta functions and the periodic orbit structure of hyperbolic dynamics, Astérisque 187-188(1990).

67] S. Patterson, "The limit set of a Fuchsian group," Acta Math. 136(1976), 241-273.

[68] M. Pollicott, "Symbolic dynamics for Smale flows," Amer. J. Math. 109(1987), 183-200.

[69] M. Pollicott and R. Sharp, "Length asymptotics in higher Teichmüller theory," Proc. A.M.S. 142(2014), 101-112.

[70] M. Pollicott and R. Sharp, "A Weil-Petersson metric on spaces of metric graphs," Geom. Ded. 172(2014), 229-244.

[71] R. Potrie and A. Sambarino, "Eigenvalues and entropy of a Hitchin representation," Invent. Math., to appear, preprint available at: http://front.math.ucdavis.edu/1411.5405

[72] D. Ruelle, "Repellers for real analytic maps," Ergodic Theory Dynamical Systems 2(1982), 99-107.

[73] D. Ruelle, Thermodynamic Formalism, Addison-Wesley, London (1978).

[74] A. Sambarino, "Quantitative properties of convex representations," Comm. Math. Helv., 89(2014), 443-488.

[75] A. Sambarino, "The orbital counting problem for hyperconvex representations," Ann. Inst. Four. 65(2015), 1755-1797.

[76] A. Sambarino, "On entropy, regularity and rigidity for convex representations of hyperbolic manifolds," Math. Annalen 364(2016), 453-483.

[77] P. Schmutz, "Die parametrisierung des Teichmüllerraumes durch geodätische Längenfunktionen," Comm. Math. Helv. 68(1993), 278-288.

[78] M. Shub, Global Stability of Dynamical Systems, Springer-Verlag, 1987.

[79] Z. Slodkowski, "Holomorphic motions and polynomial hulls," Proc. A.M.S. 111(1991), 347355.

[80] P. Storm, "Hyperbolic convex cores and simplicial volume," Duke Math. J. 140(2007), 281319

[81] D. Sullivan, "The density at infinity of a discrete group of hyperbolic motions," Inst. Hautes Études Sci. Publ. Math. 50(1979), 171-202.

[82] S. Tapie, "A variation formula for the topological entropy of convex-cocompact manifolds," Erg. Thy. Dynam. Sys. 31(2011), 1849-1864.

[83] W. Thurston, "Hyperbolic geometry and 3-manifolds," in Low-Dimensional Topology (Bangor, 1979), Cambridge University Press, 1982, 9-25.

[84] W.P. Thurston, "Hyperbolic structures on 3-manifolds, I: deformation of acylindrical manifolds," Annals of Math. 124(1986), 203-246.

[85] A. Tromba, "On a natural algebraic affine connection on the space of almost complex structures and the curvature of Teichmüller space with respect to its Weil-Petersson metric," Manu. Math. 56(1986), 475-497. 
BRIDGEMAN, CANARY, AND SAMBARINO

[86] M. Wolf, "The Teichmüller theory of harmonic maps," J. Diff. Geom. 29(1989), 449-479.

[87] M. Wolf, "The Weil-Petersson Hessian of length on Teichmüller space," J. Diff. Geom. 91(2012), 129-169.

[88] S. Wolpert, "Noncompleteness of the Weil-Petersson metric for Teichmüller space," Pac. J. Math. 61(1975), 573-577.

[89] S. Wolpert, "Chern forms and the Riemann tensor for the moduli space of curves," Invent. Math 85(1986), 119-145.

[90] S. Wolpert, "Thurston's Riemannian metric for Teichmüller space," J. Diff. Geom. 23(1986), 143-174.

[91] S. Wolpert, "Geometry of the Weil-Petersson completion of Teichmüller space," in Surveys in differential geometry, Vol. VIII (Boston, MA, 2002), Int. Press of Boston, 2003, 357-393.

[92] B. Xu, "Incompleteness of pressure metric on Teichmüller space of a bordered surface," preprint, available at: http://front.math.ucdavis.edu/1608.03937

[93] C. Yue, "The ergodic theory of discrete isometry groups on manifolds of variable negative curvature," Trans. A.M.S. 348(1996), 4965-5005.

[94] T. Zhang, "The degeneration of convex RP ${ }^{2}$ structures on surfaces," Proc. L.M.S. 111(2015), 967-1012.

[95] T. Zhang, "Degeneration of Hitchin sequences along internal sequences," G.A.F.A. 25(2015), $1588-1645$.

Boston College, Chestnut Hill, MA 02467 USA

University of Michigan, Ann Arbor, MI 41809 USA

Université Pierre et Marie Curie (Paris Vi), 75005 Paris France 